\title{
Phenomenological theory of the s-wave state in superconductors without an inversion center
}

\author{
Journal Article \\ Author(s): \\ Frigeri, P.A.; Agterberg, D.F.; Milat, I.; Sigrist, M. \\ Publication date: \\ 2006-12 \\ Permanent link: \\ https://doi.org/10.3929/ethz-b-000035485 \\ Rights / license: \\ In Copyright - Non-Commercial Use Permitted
}

Originally published in:

The European Physical Journal B 54(4), https://doi.org/10.1140/epjb/e2007-00019-5 


\title{
Phenomenological theory of the s-wave state in superconductors without an inversion center
}

\author{
P.A. Frigeri ${ }^{1}$, D.F. Agterberg ${ }^{2}$, I. Milat ${ }^{1}$, and M. Sigrist ${ }^{1, a}$ \\ 1 Theoretische Physik ETH-Hönggerberg, 8093 Zürich, Switzerland \\ 2 Department of Physics, University of Wisconsin-Milwaukee, Milwaukee, WI 53201, USA
}

Received 22 October 2006

Published online 19 January 2007 - (C) EDP Sciences, Società Italiana di Fisica, Springer-Verlag 2007

\begin{abstract}
In materials without an inversion center of symmetry the spin degeneracy of the conducting band is lifted by an antisymmetric spin orbit coupling (ASOC). Under such circumstances, spin and parity cannot be separately used to classify the Cooper pairing states. Consequently, the superconducting order parameter is generally a mixture of spin singlet and triplet pairing states. In this paper we investigate the structure of the order parameter and its response to disorder for the most symmetric pairing state $\left(A_{1}\right)$. Using the example of the heavy Fermion superconductor $\mathrm{CePt}_{3} \mathrm{Si}$, we determine characteristic properties of the superconducting instability. Depending on the type of the pairing interaction, the gap function is characterized by the presence of line nodes. We show that this line nodes move in general upon temperature. Such nodes would be essential to explain recent low-temperature data of thermodynamic quantities such as the NMR- $T_{1}^{-1}$, London penetration depth, and heat conductance. Moreover, we study the effect of (non-magnetic) impurity on the superconducting state.
\end{abstract}

PACS. 74.20.-z Theories and models of superconducting state - 71.18.+y Fermi surface: calculations and measurements; effective mass, g factor

\section{Introduction}

Early studies of superconductivity in materials without inversion symmetry addressed two-dimensional systems, such as thin films, interfaces, and surfaces [1-4]. The recent discovery of the non-centrosymmetric heavy Fermion superconductor $\mathrm{CePt}_{3} \mathrm{Si}[5]$ has drawn attention to bulk materials. Particular interest arose with the observation that the upper critical field of $\mathrm{CePt}_{3} \mathrm{Si}$ exceeds the paramagnetic limiting field considerably. The simplest interpretation of this finding is in terms of spin triplet pairing. However, this is in conflict with the common belief that the absence of an inversion center prevents electrons from forming spin-triplet pairs [7]. Indeed, inversion together with time reversal are the key symmetries required for Cooper pairing. In a time reversal invariant system, the lack of inversion symmetry is connected with the presence of an antisymmetric spin-orbit coupling (ASOC). This can be represented in the single-particle Hamiltonian by a term of the general form

$$
\alpha \boldsymbol{g}_{k} \cdot \boldsymbol{S}
$$

where the vector function $\boldsymbol{g}_{\boldsymbol{k}}$ is odd in $\boldsymbol{k}\left(\boldsymbol{g}_{\boldsymbol{k}}=-\boldsymbol{g}_{-\boldsymbol{k}}\right)$ and $\alpha$ denotes the coupling strength [6]. The ASOC is indeed detrimental for most spin triplet pairing states as noted

\footnotetext{
a e-mail: sigrist@itp.phys.ethz.ch
}

by Anderson [7]. However, it was also found that triplet states whose $\boldsymbol{d}$-vector lies parallel to $\boldsymbol{g}_{\boldsymbol{k}}$ would nevertheless be stable. Such a spin triplet state has the full symmetry of the crystal point group, we will therefore call it the $S$-triplet state from now on.

The interpretation of the absence of paramagnetism in terms of spin triplet pairing is not unique. Paramagnetic limiting is also drastically reduced for spin singlet states in the presence of ASOC $[8,6,9-12]$. In fact, the absence of the inversion symmetry leads to a breakdown of the strict classification into even-parity spin-singlet and odd-parity spin-triplet pairing; these states are mixed, resulting in a state containing both components [1-3]. Since all but one spin-triplet states are suppressed by strong enough ASOC (i.e. $\alpha \gg k_{B} T_{c}$, which is usually the case), we concentrate here on the stable $S$-triplet state. This "high-symmetry" state mixes with the " $s$-wave" spin-singlet state (which also has the full symmetry of the crystal point group), since both of them belong to the same trivial $A_{1}$ representation of $C_{4 v}$, the generating point group in $\mathrm{CePt}_{3} \mathrm{Si}$. We will call this combined phase the " $s$-wave state".

The presence of ASOC leads to a splitting of the electron bands by lifting the spin degeneracy. Thus, the discussion of the superconductivity is in some sense a two-band problem in this case. Assuming that superconductivity is restricted to a single band the basis function for irreducible representations of point group $C_{4 v}$ have 
already been determined by various groups [13-15]. These studies found that that the quasiparticle gap for the (most symmetric) $A_{1}$ state would have the form $\Delta \propto k_{x}^{2}+k_{y}^{2}+c k_{z}^{2}$ which is nodeless in general. In contrast to these earlier works, we will examine here the full two-band situation. We also show how the symmetry properties of the pairing interaction and the distribution of the density of states on the two Fermi surfaces can influence the form of the pairing state and, in particular, can introduce (accidental) line nodes in the quasiparticle gap which are not dictated by symmetry. We also show how these (accidental) line nodes move in momentum space when varying the temperature. It is also interesting to see how the $s$-wave state is affected by non-magnetic impurities. These properties could be important to obtain information on the complex structure of the pairing state in this material.

\section{Model with antisymmetric spin-orbit coupling}

The basic model used to describe the conduction electrons in crystals without an inversion center can be written as

$$
\mathcal{H}_{0}=\sum_{\boldsymbol{k}, s, s^{\prime}}\left[\xi_{\boldsymbol{k}} \sigma_{0}+\alpha \boldsymbol{g}_{\boldsymbol{k}} \cdot \boldsymbol{\sigma}\right]_{s s^{\prime}} c_{\boldsymbol{k}_{s}}^{\dagger} c_{\boldsymbol{k}_{s^{\prime}}}
$$

where $c_{\boldsymbol{k}_{s}}^{\dagger}\left(c_{\boldsymbol{k}_{s}}\right)$ creates (annihilates) an electron with wave vector $\boldsymbol{k}$ and spin $s, \hat{\boldsymbol{\sigma}}=\left(\hat{\sigma}_{x}, \hat{\sigma}_{y}, \hat{\sigma}_{z}\right)$ is the vector of Pauli matrices and $\hat{\sigma}_{0}$ is the unit matrix [6]. The band energy $\xi_{\boldsymbol{k}}=\epsilon_{\boldsymbol{k}}-\mu$ is measured relative to the chemical potential $\mu$. The antisymmetric spin-orbit coupling (ASOC) term $\alpha \boldsymbol{g}_{\boldsymbol{k}} \cdot \boldsymbol{\sigma}$ is different from zero only for crystals without an inversion center and can be derived microscopically by considering the relativistic corrections to the interaction of the electrons with the ionic potential $[9,16]$. In strongly correlated materials, as for instance in heavy Fermion, we expect $\alpha$ to be strongly renormalize from its "bare" value. For qualitative studies, it is sufficient to deduce the structure of the $\boldsymbol{g}$-vector from symmetry arguments [6] and to treat $\alpha$ as a parameter. We set $\left\langle\boldsymbol{g}_{\boldsymbol{k}}^{2}\right\rangle=1$, where \langle\rangle denotes the average over the Fermi surface. The ASOC term lifts the spin degeneracy by generating two bands with different spin structure. The normal state Green's function,

$$
\hat{G}_{0}\left(\boldsymbol{k}, i \omega_{n}\right)=G_{+}^{0}\left(\boldsymbol{k}, i \omega_{n}\right) \hat{\sigma}_{0}+\left(\hat{\boldsymbol{g}}_{\boldsymbol{k}} \cdot \hat{\boldsymbol{\sigma}}\right) G_{-}^{0}\left(\boldsymbol{k}, i \omega_{n}\right),
$$

with

$$
G_{ \pm}^{0}\left(\boldsymbol{k}, i \omega_{n}\right)=\frac{1}{2}\left[\frac{1}{i \omega_{n}-\xi_{\boldsymbol{k}}-\alpha\left|\boldsymbol{g}_{\boldsymbol{k}}\right|} \pm \frac{1}{i \omega_{n}-\xi_{\boldsymbol{k}}+\alpha\left|\boldsymbol{g}_{\boldsymbol{k}}\right|}\right],
$$

and $\hat{\boldsymbol{g}}_{\boldsymbol{k}}=\boldsymbol{g}_{\boldsymbol{k}} /\left|\boldsymbol{g}_{\boldsymbol{k}}\right|\left(|\boldsymbol{g}|=\sqrt{\boldsymbol{g}^{2}}\right)$, can be diagonalized into the components corresponding to the two bands, using the unitary transformation

$$
\hat{U}(\boldsymbol{k})=\cos \left(\theta_{\boldsymbol{k}} / 2\right)-i \sin \left(\theta_{\boldsymbol{k}} / 2\right)\left(\cos \phi_{\boldsymbol{k}} \hat{\sigma}_{y}-\sin \phi_{\boldsymbol{k}} \hat{\sigma}_{x}\right),
$$

where $\boldsymbol{g}_{\boldsymbol{k}}=\left|\boldsymbol{g}_{\boldsymbol{k}}\right|\left(\sin \theta_{\boldsymbol{k}} \cos \phi_{\boldsymbol{k}}, \sin \theta_{\boldsymbol{k}} \sin \phi_{\boldsymbol{k}}, \cos \theta_{\boldsymbol{k}}\right)$ defines the angles $\theta_{\boldsymbol{k}}$ and $\phi_{\boldsymbol{k}}$. This allows us to express,

$$
\hat{G}_{0}\left(\boldsymbol{k}, i \omega_{n}\right)=G_{1}^{0}\left(\boldsymbol{k}, i \omega_{n}\right) \hat{\sigma}_{1}(\boldsymbol{k})+G_{2}^{0}\left(\boldsymbol{k}, i \omega_{n}\right) \hat{\sigma}_{2}(\boldsymbol{k}),
$$
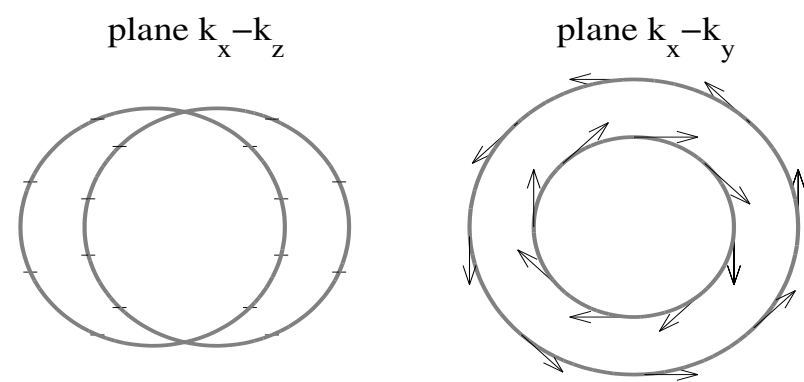

Fig. 1. Fermi surfaces for $\boldsymbol{g}_{\boldsymbol{k}} \propto\left(k_{y},-k_{x}, 0\right)$ as in $\mathrm{CePt}_{3} \mathrm{Si}$. The arrows show the structure of the quasi-particle spin. Only along the $z$-axis the spin degeneracy is preserved.

through the two Green's functions,

$$
G_{1,2}^{0}\left(\boldsymbol{k}, i \omega_{n}\right)=\frac{1}{i \omega_{n}-\xi_{1,2}(\boldsymbol{k})}
$$

where the quasi-particle bands $\xi_{1,2}(\boldsymbol{k}) \equiv \xi_{\boldsymbol{k}} \pm \alpha\left|\boldsymbol{g}_{\boldsymbol{k}}\right|$ are split by the presence of the ASOC. The spin structure in the bands is described by $2 \times 2$ matrixes

$$
\begin{aligned}
\sigma_{\lambda, \mu}^{1}(\boldsymbol{k}) & \equiv U_{\lambda, 1}(\boldsymbol{k}) U_{1, \mu}^{\dagger}(\boldsymbol{k})=1 / 2\left(\sigma_{0}+\left(\hat{\boldsymbol{g}}_{\boldsymbol{k}} \cdot \boldsymbol{\sigma}\right)\right)_{\lambda, \mu} \\
\sigma_{\lambda, \mu}^{2}(\boldsymbol{k}) & \equiv U_{\lambda, 2}(\boldsymbol{k}) U_{2, \mu}^{\dagger}(\boldsymbol{k})=1 / 2\left(\sigma_{0}-\left(\hat{\boldsymbol{g}}_{\boldsymbol{k}} \cdot \boldsymbol{\sigma}\right)\right)_{\lambda, \mu}
\end{aligned}
$$

which act as projection operators in spin space. Since the spins of quasiparticles in the two bands have opposite directions for a given momentum $\boldsymbol{k}$, the matrices satisfy the conditions,

$$
\begin{aligned}
\hat{\sigma}_{1}(\boldsymbol{k}) \hat{\sigma}_{2}(\boldsymbol{k}) & =0 \\
\hat{\sigma}_{i}^{2}(\boldsymbol{k}) & =\hat{\sigma}_{i}(\boldsymbol{k}), \quad(i=1,2)
\end{aligned}
$$

and are reciprocal under the inversion operation,

$$
\hat{\sigma}_{1}(-\boldsymbol{k})=\hat{\sigma}_{2}(\boldsymbol{k})
$$

Symmetry considerations lead to the following form for $\boldsymbol{g}_{\boldsymbol{k}}$ in $\mathrm{CePt}_{3} \mathrm{Si}$ in lowest order expansion in the wave vector $\boldsymbol{k}: \boldsymbol{g}_{\boldsymbol{k}} \propto\left(k_{y},-k_{x}, 0\right)$. The resulting spin structure is visualized in Figure 1, where we assume for simplicity that the original Fermi surface is spherical.

The pairing interaction is generally given by

$$
\mathcal{H}_{\text {pair }}=\frac{1}{2} \sum_{\boldsymbol{k}, \boldsymbol{k}^{\prime}} \sum_{s, s^{\prime}} V_{s_{1} s_{2}, s_{2}^{\prime} s_{1}^{\prime}}\left(\boldsymbol{k}, \boldsymbol{k}^{\prime}\right) c_{\boldsymbol{k}_{s_{1}}^{\dagger}} c_{-\boldsymbol{k}_{s_{2}}}^{\dagger} c_{-\boldsymbol{k}^{\prime} s_{2}^{\prime}} c_{\boldsymbol{k}^{\prime} s_{1}^{\prime}}
$$

where $V_{s_{1} s_{2}, s_{2}^{\prime} s_{1}^{\prime}}\left(\boldsymbol{k}, \boldsymbol{k}^{\prime}\right)$ is the pairing potential with the symmetry properties,

$$
\begin{aligned}
V_{s_{1} s_{2}, s_{2}^{\prime} s_{1}^{\prime}}\left(\boldsymbol{k}, \boldsymbol{k}^{\prime}\right) & =-V_{s_{2} s_{1}, s_{2}^{\prime} s_{1}^{\prime}}\left(-\boldsymbol{k}, \boldsymbol{k}^{\prime}\right) \\
& =-V_{s_{1} s_{2}, s_{1}^{\prime} s_{2}^{\prime}}\left(\boldsymbol{k},-\boldsymbol{k}^{\prime}\right) \\
& =V_{s_{1}^{\prime} s_{2}^{\prime}, s_{2} s_{1}}\left(\boldsymbol{k}^{\prime}, \boldsymbol{k}\right) .
\end{aligned}
$$

In unconventional superconductors, the state of knowledge on the nature of the pairing potential is rather limited. However, in the context of the BCS theory, we can 
deduce the form of the attractive interaction using symmetry arguments. More precisely, it is possible to build up the effective pairing potential starting from the form of the order parameter under investigation $[17,18]$.

We are interested here in the study of the most symmetric superconducting state. In the absence of the ASOC the system is inversion symmetric in $\boldsymbol{k}$-space and the interaction factorizes in an orbital and a spin part. The most symmetric superconducting state is in this case the conventional $s$-wave spin-singlet state. The corresponding superconducting order parameter is frequently represented by the $2 \times 2$ matrix $\psi(\boldsymbol{k}) i \sigma_{y}$, where $\psi(\boldsymbol{k})$ is a basis state of trivial representation $A_{1 g}$ of the inversion symmetric point group $G$, which can be considered in the simplest case as a complex constant $\psi(\boldsymbol{k}) \equiv \psi$.

When the ASOC is turned on, the point group is reduced to the non-inversion symmetric subgroup $G^{\prime}$, whose trivial representation $A_{1}$ does not have a definite parity. In fact $A_{1}$ is compatible with both odd and even representations of $G$, i.e. with both $\left\{A_{1 g}, \Gamma_{u}\right\}$, where $\Gamma_{u}$ is an odd parity irreducible representation in $G$, for which $\boldsymbol{g}_{\boldsymbol{k}}$ is a basis state [21].

In other words, the symmetries of the non-inversion symmetric point group $G^{\prime}$ do not allow us to distinguish between the $s$-wave spin-singlet state and the spin-triplet state characterized by the order parameter $d\left(\boldsymbol{g}_{\boldsymbol{k}} \cdot \hat{\boldsymbol{\sigma}}\right) \hat{\sigma}_{y}$. This is because the inversion element is missing in the point group $G^{\prime}$, thus the parity of the superconducting state is no longer a good quantum number. The superconducting state involves both spin-singlet $\psi$ and spin-triplet $d$ components. The order parameter for the "highestsymmetry" state reads

$$
\hat{\Delta}(\boldsymbol{k})=i\left\{\psi+d\left(\boldsymbol{g}_{\boldsymbol{k}} \cdot \hat{\boldsymbol{\sigma}}\right)\right\} \hat{\sigma}_{y} .
$$

Since both spin-singlet and spin-triplet components belongs to the same irreducible representation $A_{1}$ of $G^{\prime}$, the spectral form of the pairing interaction relevant for the realisation of this superconducting state involves, in its general form, three different terms

$$
\begin{aligned}
& V_{s_{1} s_{2}, s_{2}^{\prime} s_{1}^{\prime}}\left(\boldsymbol{k}, \boldsymbol{k}^{\prime}\right)=\frac{V}{2}\left\{e_{s} \hat{\tau}_{s_{1} s_{2}} \hat{\tau}_{s_{2}^{\prime} s_{1}^{\prime}}^{\dagger}\right. \\
&+e_{t}\left[\left(\boldsymbol{g}_{\boldsymbol{k}} \cdot \hat{\boldsymbol{\tau}}\right)_{s_{1} s_{2}}\left(\boldsymbol{g}_{\boldsymbol{k}^{\prime}} \cdot \hat{\boldsymbol{\tau}}\right)_{s_{2}^{\prime} s_{1}^{\prime}}^{\dagger}\right] \\
&\left.+e_{m}\left[\left(\boldsymbol{g}_{\boldsymbol{k}} \cdot \hat{\boldsymbol{\tau}}\right)_{s_{1} s_{2}} \hat{\tau}_{s_{2}^{\prime} s_{1}^{\prime}}^{\dagger}+\hat{\tau}_{s_{1} s_{2}}\left(\boldsymbol{g}_{\boldsymbol{k}^{\prime}} \cdot \hat{\boldsymbol{\tau}}\right)_{s_{2}^{\prime} s_{1}^{\prime}}^{\dagger}\right]\right\},
\end{aligned}
$$

where $\hat{\tau}_{s_{1} s_{2}}=\left(i \hat{\sigma}^{y}\right)_{s_{1} s_{2}}$, and, $\hat{\boldsymbol{\tau}}_{s_{1} s_{2}}=\left(i \boldsymbol{\sigma} \hat{\sigma}^{y}\right)_{s_{1} s_{2}}$. To avoid ambiguity we set $V>0$, and $e_{s}^{2}+e_{t}^{2}+e_{m}^{2}=1$.

The first two terms of equation (14) are diagonal in the conventional $s$-wave pairing channel and in the $S$-triplet pairing channel [6], respectively. The last term, which describes the scattering of Cooper pairs between the two channels, is allowed by the fact that the spin-singlet $\psi$ and the spin-triplet $d$ components belongs to the same irreducible representation of the point groups. This is a result of the absence of inversion symmetry.

The microscopic origin of pairing interaction is not the subject of this phenomenological paper. However in Appendix A we show that the mixed pairing interaction term $e_{m}$ can be generated by the Dzyaloshinskii-Moriya type of interaction $[19,20]$ for both a weakly interacting Fermi liquid and for a Hubbard model near half filling. Any quantitative evaluation of this term is out of the scope of this paper. Moreover, the main results of our work do not depend on the magnitude of this term.

We study the superconductivity by means of Gor'kov equations which are formally analogous to those obtained for systems with an inversion symmetry [22]

$$
\begin{aligned}
\hat{G}_{0}^{-1}\left(\boldsymbol{k}, i \omega_{n}\right) \hat{G}\left(\boldsymbol{k}, i \omega_{n}\right)+\hat{\Delta}(\boldsymbol{k}) \hat{F}^{\dagger}\left(\boldsymbol{k}, i \omega_{n}\right) & =\hat{\sigma}_{0} \\
\hat{G}_{0}^{-1^{\top}}\left(-\boldsymbol{k},-i \omega_{n}\right) \hat{F}^{\dagger}\left(\boldsymbol{k}, i \omega_{n}\right)-\hat{\Delta}^{\dagger}(\boldsymbol{k}) \hat{G}\left(\boldsymbol{k}, i \omega_{n}\right) & =0 \\
\hat{G}_{0}^{-1}\left(\boldsymbol{k}, i \omega_{n}\right) \hat{F}\left(\boldsymbol{k}, i \omega_{n}\right)-\hat{\Delta}(\boldsymbol{k}) \hat{G}^{\top}\left(-\boldsymbol{k},-i \omega_{n}\right) & =0,
\end{aligned}
$$

where $\hat{G}$ and $\hat{F}$ denotes the regular and the anomalous Green's functions, respectively. The two-band structure of the normal state is more conveniently handled if we use the Green's functions $G_{1,2}$ and $F_{1,2}$, as is easily obtained by the unitary transformation (5),

$$
\begin{aligned}
\left(\begin{array}{cc}
G_{1} & 0 \\
0 & G_{2}
\end{array}\right) & =\hat{U}^{\dagger}(\boldsymbol{k}) \hat{G} \hat{U}(\boldsymbol{k}) \\
-\left(\begin{array}{cc}
F_{1} e^{-i \phi} \boldsymbol{k} & 0 \\
0 & F_{2} e^{i \phi} \boldsymbol{k}
\end{array}\right) & =\hat{U}^{\dagger}(\boldsymbol{k}) \hat{F} \hat{U}^{*}(-\boldsymbol{k}) .
\end{aligned}
$$

The phase factor $-e^{\mp i \phi} \boldsymbol{k}$ is introduced to cancel the phase dependence of the anomalous Green's functions on the two bands [14].

The particular form of $\hat{\Delta}(\boldsymbol{k})$, equation (13), prevents the existence of inter-band terms in the Gorkov equations

$$
\begin{array}{r}
\left\{G_{1,2}^{0}\left(\boldsymbol{k}, i \omega_{n}\right)\right\}^{-1} G_{1,2}\left(\boldsymbol{k}, i \omega_{n}\right) \\
+\Delta_{1,2}(\boldsymbol{k}) F_{1,2}^{\dagger}\left(\boldsymbol{k}, i \omega_{n}\right)=1 \\
\left\{G_{1,2}^{0}\left(-\boldsymbol{k},-i \omega_{n}\right)\right\}^{-1} F_{1,2}^{\dagger}\left(\boldsymbol{k}, i \omega_{n}\right) \\
-\Delta_{1,2}^{*}(\boldsymbol{k}) G_{1,2}\left(\boldsymbol{k}, i \omega_{n}\right)=0,
\end{array}
$$

where, in this case,

$$
\Delta_{1,2}(\boldsymbol{k})=\left(\psi \pm d\left|\boldsymbol{g}_{\boldsymbol{k}}\right|\right)
$$

Thus, the Gor'kov equations are diagonal in the band index. The solution of the two-band Gorkov equations is given by

$$
G_{1,2}\left(\boldsymbol{k}, i \omega_{n}\right)=-\frac{i \omega_{n}+\xi_{1,2}}{\left(\omega_{n}^{2}+\left|\Delta_{1,2}\right|^{2}+\xi_{1,2}^{2}\right)},
$$

and

$$
F_{1,2}\left(\boldsymbol{k}, i \omega_{n}\right)=\frac{\Delta_{1,2}}{\left(\omega_{n}^{2}+\left|\Delta_{1,2}\right|^{2}+\xi_{1,2}^{2}\right)} .
$$

Going back to the spin basis, we find

$$
\begin{aligned}
& \hat{G}\left(\boldsymbol{k}, i \omega_{n}\right)=G_{1}\left(\boldsymbol{k}, i \omega_{n}\right) \hat{\sigma}_{1}(\boldsymbol{k})+G_{2}\left(\boldsymbol{k}, i \omega_{n}\right) \hat{\sigma}_{2}(\boldsymbol{k}) \\
& \hat{F}\left(\boldsymbol{k}, i \omega_{n}\right)=\left\{F_{1}\left(\boldsymbol{k}, i \omega_{n}\right) \hat{\sigma}_{1}(\boldsymbol{k})+F_{2}\left(\boldsymbol{k}, i \omega_{n}\right) \hat{\sigma}_{2}(\boldsymbol{k})\right\} i \hat{\sigma}_{y}
\end{aligned}
$$


The gap functions of the two bands are obtained by solving the self-consistent equation

$$
\Delta_{\alpha}(\boldsymbol{k})=-k_{B} T \int \frac{d \boldsymbol{k}^{\prime}}{(2 \pi)^{3}} \sum_{n, \beta} V_{\boldsymbol{k}, \boldsymbol{k}^{\prime}}^{\alpha, \beta} F_{\beta}\left(\boldsymbol{k}^{\prime}, i \omega_{n}\right) .
$$

The corresponding pairing interaction is determined by

$$
\begin{aligned}
V_{\boldsymbol{k}, \boldsymbol{k}^{\prime}}^{\alpha, \beta}= & \sum_{\gamma, \delta} \sum_{s_{i}, s_{i}^{\prime}} D_{\alpha, \gamma}^{*}(\boldsymbol{k}) U_{s_{1} \gamma}^{*}(-\boldsymbol{k}) \hat{U}_{\gamma s_{2}}^{\dagger}(\boldsymbol{k}) V_{s_{1} s_{2}, s_{2}^{\prime} s_{1}^{\prime}}\left(\boldsymbol{k}, \boldsymbol{k}^{\prime}\right) \\
& \times \hat{U}_{s_{2}^{\prime} \delta}\left(\boldsymbol{k}^{\prime}\right) \hat{U}_{\delta s_{1}^{\prime}}^{\top}\left(-\boldsymbol{k}^{\prime}\right) D_{\delta, \beta}\left(\boldsymbol{k}^{\prime}\right)
\end{aligned}
$$

with $\hat{D}\left(\boldsymbol{k}^{\prime}\right)=\operatorname{diag}\left(-\exp \left(-i \phi_{\boldsymbol{k}^{\prime}}\right),-\exp \left(i \phi_{\boldsymbol{k}^{\prime}}\right)\right)$. Using (14) it follows

$$
\begin{aligned}
\hat{V}_{\boldsymbol{k}, \boldsymbol{k}^{\prime}}= & \frac{V}{2}\left\{\left[e_{s}+e_{t}\left|\boldsymbol{g}_{\boldsymbol{k}}\right|\left|\boldsymbol{g}_{\boldsymbol{k}^{\prime}}\right|\right] \hat{\sigma}_{0}+\left[e_{s}-e_{t}\left|\boldsymbol{g}_{\boldsymbol{k}}\right|\left|\boldsymbol{g}_{\boldsymbol{k}^{\prime}}\right|\right] \hat{\sigma}_{x}\right. \\
& \left.-e_{m}\left[\left|\boldsymbol{g}_{\boldsymbol{k}}\right|+\left|\boldsymbol{g}_{\boldsymbol{k}^{\prime}}\right|\right] \hat{\sigma}_{z}-i e_{m}\left[\left|\boldsymbol{g}_{\boldsymbol{k}}\right|-\left|\boldsymbol{g}_{\boldsymbol{k}^{\prime}}\right|\right] \hat{\sigma}_{y}\right\}
\end{aligned}
$$

The evaluation of $\boldsymbol{\Delta}=(\psi, d)$ is done by solving equation (22). We approximate the sum over $\boldsymbol{k}$ by $\sum_{\boldsymbol{k}} \rightarrow$ $N_{0} \int d \Omega / 4 \pi \int d \xi$. After having performed the integral over $\xi$, the gap equation reads

$$
\frac{1}{N_{0} V} \boldsymbol{\Delta}=\pi k_{B} T \sum_{\omega_{n}>-\epsilon_{c}}^{\epsilon_{c}} \hat{Q}\left(i \omega_{n}\right) \boldsymbol{\Delta}
$$

with

$$
\begin{aligned}
\hat{Q}= & {\left[Q_{1}\left(i \omega_{n}\right)\left(\begin{array}{cc}
-e_{s} & 0 \\
e_{m} & 0
\end{array}\right)+Q_{2}\left(i \omega_{n}\right)\left(\begin{array}{cc}
e_{m} & -e_{s} \\
-e_{t} & e_{m}
\end{array}\right)\right.} \\
& \left.+Q_{3}\left(i \omega_{n}\right)\left(\begin{array}{cc}
0 & e_{m} \\
0 & -e_{t}
\end{array}\right)\right]
\end{aligned}
$$

where we introduced the functions

$$
\begin{aligned}
& Q_{1} \equiv \frac{1}{2}\left\langle\frac{\left(1+\delta_{N}\right)}{\sqrt{\omega_{n}^{2}+|\psi+d| \boldsymbol{g}_{\boldsymbol{k}} \|^{2}}}+\frac{\left(1-\delta_{N}\right)}{\sqrt{\omega_{n}^{2}+\left.|\psi-d| \boldsymbol{g}_{\boldsymbol{k}}\right|^{2}}}\right\rangle_{\boldsymbol{k}} \\
& Q_{2} \equiv \frac{1}{2}\left\langle\frac{\left(1+\delta_{N}\right)\left|\boldsymbol{g}_{\boldsymbol{k}}\right|}{\sqrt{\omega_{n}^{2}+|\psi+d| \boldsymbol{g}_{\boldsymbol{k}} \|^{2}}}-\frac{\left(1-\delta_{N}\right)\left|\boldsymbol{g}_{\boldsymbol{k}}\right|}{\sqrt{\omega_{n}^{2}+\left.|\psi-d| \boldsymbol{g}_{\boldsymbol{k}}\right|^{2}}}\right\rangle_{\boldsymbol{k}} \\
& Q_{3} \equiv \frac{1}{2}\left\langle\frac{\left(1+\delta_{N}\right)\left|\boldsymbol{g}_{\boldsymbol{k}}\right|^{2}}{\sqrt{\omega_{n}^{2}+|\psi+d| \boldsymbol{g}_{\boldsymbol{k}} \|^{2}}}+\frac{\left(1-\delta_{N}\right)\left|\boldsymbol{g}_{\boldsymbol{k}}\right|^{2}}{\sqrt{\omega_{n}^{2}+\left.|\psi-d| \boldsymbol{g}_{\boldsymbol{k}}\right|^{2}}}\right\rangle_{\boldsymbol{k}}
\end{aligned}
$$

where \langle\rangle$_{\boldsymbol{k}}$ denotes the integral over the Fermi surface $\int d \Omega_{\boldsymbol{k}} / 4 \pi$. The parameter $\delta_{N}$ fixes the distribution of the DOS at the Fermi level of the two bands, $N_{1}=N_{0}\left(1+\delta_{N}\right)$ and $N_{2}=N_{0}\left(1-\delta_{N}\right)$.

\section{Characterization of the superconducting instability}

The critical temperature and the basic structure of the gap function $\boldsymbol{\Delta}=(\psi, d)$, which characterizes the superconducting instability, follow from the solution of the linearized form of the self-consistent equation (25). We find

$$
\frac{1}{N_{0} V} \boldsymbol{\Delta}=f_{1}\left(\epsilon_{c}, k_{B} T\right) \hat{Q}^{l} \boldsymbol{\Delta}
$$

with

$$
f_{1}\left(\epsilon_{c}, k_{B} T\right)=\ln \left(4 \gamma \epsilon_{c} / 2 \pi k_{B} T\right)
$$

and

$$
\hat{Q}^{l} \equiv\left[\left(\begin{array}{cc}
-e_{s} & e_{m} \\
e_{m} & -e_{t}
\end{array}\right)+\delta_{N}\left\langle\left|\boldsymbol{g}_{\boldsymbol{k}}\right|\right\rangle\left(\begin{array}{cc}
e_{m} & -e_{s} \\
-e_{t} & e_{m}
\end{array}\right)\right] .
$$

where we have used the Euler's constant $\gamma=C \approx 0.577$. For simplicity, we have assumed the same cut-off energy $\epsilon_{c}$ for both bands.

Equation (28) has a non-trivial solution, if at least one of the eigenvalues $\lambda_{i}$ of the matrix $\hat{Q}^{l}$ is positive. In this case, the critical temperature follows the standard BCS relation $k_{B} T_{c}=2 \epsilon_{c} \gamma / \pi \exp \left(-1 /\left(N_{0} V \lambda^{\prime}\right)\right)$, where $\lambda^{\prime}=$ $\max _{i}\left(\lambda_{i}\right)>0$. Furthermore, the nucleating form of the gap function follows from $\hat{Q}^{l} \boldsymbol{\Delta}^{\prime}=\lambda^{\prime} \boldsymbol{\Delta}^{\prime}$.

In this context, we introduce the term dominant channel to denote the channel responsible for the superconducting transition, and we call subdominant the other channel characterized by $\lambda^{\prime \prime}=\min _{i}\left(\lambda_{i}\right)$ and $\hat{Q}^{l} \boldsymbol{\Delta}^{\prime \prime}=$ $\lambda^{\prime \prime} \boldsymbol{\Delta}^{\prime \prime}$.

Solving the eigenstate problem $\hat{Q}^{l} \boldsymbol{\Delta}_{i}=\lambda_{i} \boldsymbol{\Delta}_{i}$, we find the sets

$$
\begin{aligned}
2 \lambda^{\prime} & =-\left(e_{s}+e_{t}\right)+2 e_{m} \delta+\Lambda \\
\boldsymbol{\Delta}^{\prime} & \propto\left(\begin{array}{c}
-\left(e_{s}-e_{t}\right)+\Lambda \\
2\left(e_{m}-e_{t} \delta\right)
\end{array}\right)
\end{aligned}
$$

and

$$
\begin{aligned}
& 2 \lambda^{\prime \prime}=-\left(e_{s}+e_{t}\right)+2 e_{m} \delta-\Lambda, \\
& \Delta^{\prime \prime} \propto\left(\begin{array}{c}
-\left(e_{s}-e_{t}\right)-\Lambda \\
2\left(e_{m}-e_{t} \delta\right)
\end{array}\right) .
\end{aligned}
$$

where

$$
\Lambda=\sqrt{\left(e_{s}-e_{t}\right)^{2}+4\left(e_{m}-e_{s} \delta\right)\left(e_{m}-e_{t} \delta\right)},
$$

and $\delta=\delta_{N}\left\langle\left|\boldsymbol{g}_{\boldsymbol{k}}\right|\right\rangle$ to simplify the notation. Our interest lies in the characterization of the instability for all possible combinations of the three components of the pairing potential (14). From equation (31), we determine the conditions for the superconducting instability and the form of the nucleating pairing state given by equation (32).

Obviously an attractive interaction either for spinsinglet or -triplet pairing or both would yield a superconducting instability. If both $e_{s}$ and $e_{t}$ are repulsive $(>0)$, the 
mixing term plays the decisive role. From equation (31) we immediately see that an instability is absent for small $\left|e_{m}\right|$ :

$$
\left|e_{m}\right|<\sqrt{e_{s} e_{t}} \text { where } e_{s}, e_{t}>0 .
$$

On the other hand, a large enough value of $\left|e_{m}\right|$ can trigger superconductivity, even with $e_{s}, e_{t}>0$. The inter-parity scattering can lower the pairing energy and generate a positive eigenvalue of the matrix $\hat{Q}^{l}$. This mechanism is formally analogous to superconductivity driven by interband Cooper pair scattering in a multi-band superconductor.

It remains to determine the form of the 2-dimensional order parameter $\Delta=(\psi, d)$ based on equation (32) which nucleates at the superconducting transition. Before we start discussing the conditions favoring different order parameter forms, we will consider the corresponding gap topologies on the two bands. To be concrete, we now discuss the case relevant for $\mathrm{CePt}_{3} \mathrm{Si}$, i.e. $\boldsymbol{g}_{\boldsymbol{k}} \propto\left(k_{y},-k_{x}, 0\right)$. The gap on the two Fermi surfaces is given by

$$
\Delta_{1,2}(\theta)=\left(\psi \pm d C_{1}|\sin (\theta)|\right),
$$

with $C_{1}=\sqrt{3 / 2}$ in the case of a spherical original Fermi surface and, $\theta$ is the polar angle in $\boldsymbol{k}$-space relative to the $z$-axis. It is easly to see from equation (36) that the gap topologies on the two bands depend only on the ratio between the spin-singlet and the spin-triplet components at the critical temperature

$$
\nu_{l} \equiv \lim _{T \rightarrow T_{c}} \frac{\psi}{d}=-\frac{\left(e_{s}-e_{t}\right)+\Lambda}{2\left(e_{m}-e_{t} \delta\right)} .
$$

More precisely we find that

$$
\left|\nu_{l}\right|\left\{\begin{array}{cc}
=0 & \text { point-nodes } \\
<C_{1} & \text { line-nodes } \perp z \text {-axis } \\
>C_{1} & \text { no nodes. }
\end{array}\right.
$$

In case of a pure spin-triplet phase $\nu_{l}=0$, the two gap functions $\Delta_{1}$ and $\Delta_{2}$ have the same amplitude and two point-nodes appear along the $z$-axis. For a strongly dominant spin-singlet phase with $\left|\nu_{l}\right|>C_{1}$, the two gap functions do not show any kind of nodes. The dominant but mixed spin-triplet phase characterazed by $0<\left|\nu_{l}\right|<C_{1}$ show the most interesting situation. In this case one of the two gap function is characterized by the presence of two line nodes perpendicular to the $z$-axis as showed in Figure 2 . Now we turn to the conditions under which the order parameter with different topologies nucleate. We expect that point nodes, which characterize the pure spin-triplet order parameter, appears if the spin-triplet potential $e_{t}$ is attractive and dominant. This is the case only if the density of states on the Fermi levels is equal to $\delta=0$ and the parity-mixing interaction $e_{m}$ is absent. For the general case with $e_{m} \neq 0$ equation (32) indicates that $e_{m}=e_{s} \delta$ is necessary to compensate the mixing of the spin-triplet with the spin-singlet pairing channels induced when the DOS are different for the two Fermi surfaces $\delta \neq 0$. If this last condition is not satisfied, $e_{m} \neq e_{s} \delta$, a spin-singlet component appears giving rise to line nodes on one of the

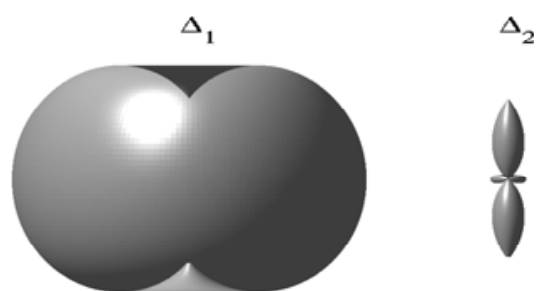

Fig. 2. The gap amplitudes $\Delta_{1,2}$ of the two non-degenerated bands for $\nu_{l}=1$.

two non-degenerates bands. We can then conclude that line nodes could appear in $\mathrm{CePt}_{3} \mathrm{Si}$ as a consequence of a dominant spin-triplet pairing interaction, $e_{t}<e_{s}$. Because we can easily assume that in general a material without an inversion center is characterized by $e_{m} \neq e_{s} \delta$.

\section{Evolution of the gaps below $T_{c}$}

To obtain the magnitude of the two-dimensional order parameter $\boldsymbol{\Delta}=(\psi, d)$ as a function of the temperature, we have to solve the self-consistent equation (25). This becomes simpler at $T=0$,

$$
\boldsymbol{\Delta}=\hat{Q}_{0}(\boldsymbol{\Delta}) \boldsymbol{\Delta}
$$

with

$$
\begin{array}{r}
\hat{Q}_{0}(\boldsymbol{\Delta})=\left\{V N _ { 0 } \left[Q_{1}^{0}(\boldsymbol{\Delta})\left(\begin{array}{rr}
-e_{s} & 0 \\
e_{m} & 0
\end{array}\right)+Q_{2}^{0}(\boldsymbol{\Delta})\left(\begin{array}{cc}
e_{m} & -e_{s} \\
-e_{t} & e_{m}
\end{array}\right)\right.\right. \\
\left.\left.+Q_{3}^{0}(\boldsymbol{\Delta})\left(\begin{array}{ll}
0 & e_{m} \\
0 & -e_{t}
\end{array}\right)\right]+\frac{1}{\lambda^{\prime}} \hat{Q}^{l}\right\},
\end{array}
$$

where $\hat{Q}^{l}$ and $\lambda^{\prime}$ have been defined in equations (30) and (31). The functions

$$
\begin{aligned}
& Q_{1}^{0} \equiv\left\langle\ln \left(\frac{\gamma}{\pi k_{B} T_{c}}\left|\Delta_{1}(\boldsymbol{k})\right|^{\frac{\left(1+\delta_{N}\right)}{2}}\left|\Delta_{2}(\boldsymbol{k})\right|^{\frac{\left(1-\delta_{N}\right)}{2}}\right)\right\rangle_{\boldsymbol{k}}, \\
& Q_{2}^{0} \equiv\left\langle\left|\boldsymbol{g}_{\boldsymbol{k}}\right| \ln \left(\left(\frac{\gamma}{\pi k_{B} T_{c}}\right)^{\delta_{N}} \frac{\left|\Delta_{1}(\boldsymbol{k})\right|^{\frac{\left(1+\delta_{N}\right)}{2}}}{\left|\Delta_{2}(\boldsymbol{k})\right|^{\frac{\left(1-\delta_{N}\right)}{2}}}\right)\right\rangle_{\boldsymbol{k}}, \\
& Q_{3}^{0} \equiv\left\langle\left|\boldsymbol{g}_{\boldsymbol{k}}\right|^{2} \ln \left(\frac{\gamma}{\pi k_{B} T_{c}}\left|\Delta_{1}(\boldsymbol{k})\right|^{\frac{\left(1+\delta_{N}\right)}{2}}\left|\Delta_{2}(\boldsymbol{k})\right|^{\frac{\left(1-\delta_{N}\right)}{2}}\right)\right\rangle_{\boldsymbol{k}}
\end{aligned}
$$

are obtained from equation (27). The eigenproblem $\lambda_{i}^{0} \boldsymbol{\Delta}_{i}^{0}=\hat{Q}_{0}\left(\boldsymbol{\Delta}_{i}^{0}\right) \boldsymbol{\Delta}_{i}^{0}$ determines the ratio $\nu_{0}=\psi_{0} / d_{0}$ between the $s$-wave spin-singlet $\psi_{0}$ and the S-triplet $d_{0}$ component at $T=0$. However, the additional condition $\lambda_{i}^{0}\left(\boldsymbol{\Delta}_{i}^{0}\right)=1$ is necessary to determine the magnitude of the two components of the order parameter.

The parameters involved in equations (25) and (39) are the three components of the pairing interaction $\left(e_{s}, e_{t}, e_{m}\right)$, the density of states on the two nondegenerate bands $\delta_{N}$ and the strength of the interaction $1 / N_{0} V$. To simplify the study of such equations, 
it is more convenient to derive the three components of the pairing interaction $\left(e_{s}, e_{t}, e_{m}\right)$ from parameters which characterize the superconducting instability. These are the ratio $\nu_{l}$, introduced in equation (37), and a second parameter $\rho$ which characterizes the subdominant channel,

$$
\rho \equiv \frac{1}{N_{0} V} \frac{\lambda^{\prime \prime}-\lambda^{\prime}}{\lambda^{\prime} \lambda^{\prime \prime}} .
$$

$\lambda^{\prime \prime}$ has been defined in equation (33). If the subdominant channel is attractive, then $\rho=\ln \left(T_{c}^{\prime \prime} / T_{c}\right)$ with

$$
k_{B} T_{c}^{\prime \prime}=2 \epsilon_{c} \gamma / \pi \exp \left(-1 /\left(N_{0} V \lambda^{\prime \prime}\right)\right),
$$

otherwise $\rho=1 / N_{0} V_{\text {eff }}$, where

$$
\frac{1}{N_{0} V_{e f f}}=\frac{1}{N_{0} V \lambda^{\prime}}-\frac{1}{N_{0} V \lambda^{\prime \prime}}
$$

defines the effective interaction in the case of a repulsive subdominant channel.

Introducing

$$
\frac{1}{\kappa} \equiv \sum_{0 \leq n<\left(\frac{\epsilon_{c}}{2 \pi k_{B} T_{c}}-\frac{1}{2}\right)} \frac{2}{2 n+1}=\frac{1}{N_{0} V \lambda^{\prime}}
$$

and through substitution of $\lambda^{\prime}$ and $\lambda^{\prime \prime}$ in equations (37, $41,43)$, we find $\left(e_{s}, e_{t}, e_{m}\right)=\left(\lambda_{s}, \lambda_{t}, \lambda_{m}\right) / \sqrt{\lambda_{s}^{2}+\lambda_{t}^{2}+\lambda_{m}^{2}}$ with

$$
\begin{aligned}
& \lambda_{s}=-\frac{\kappa\left(1+\nu_{l}^{2}+2 \nu_{l} \delta+\kappa \nu_{l}^{2}\left(\delta^{2}-1\right) \rho\right)}{\left(1+\nu_{l}^{2}+2 \nu_{l} \delta\right)\left(\delta^{2}-1\right)(\kappa \rho-1)} \\
& \lambda_{t}=-\frac{\kappa\left(1+\nu_{l}^{2}+2 \nu_{l} \delta+\kappa\left(\delta^{2}-1\right) \rho\right)}{\left(1+\nu_{l}^{2}+2 \nu_{l} \delta\right)\left(\delta^{2}-1\right)(\kappa \rho-1)} \\
& \lambda_{m}=\frac{\kappa\left(\kappa \nu_{l}\left(\delta^{2}-1\right) \rho-\delta\left(1+\nu_{l}^{2}+2 \nu_{l} \delta\right)\right)}{\left(1+\nu_{l}^{2}+2 \nu_{l} \delta\right)\left(\delta^{2}-1\right)(\kappa \rho-1)} .
\end{aligned}
$$

\section{The case $\lambda^{\prime \prime}=0$}

We start the discussion considering the case where the subdominant channel is inactive, $\lambda^{\prime \prime}=0$. In this case, the interaction parameters $e_{s}, e_{t}$, and, $e_{m}$ lie on a line given by

$$
\left|e_{m}\right|=\sqrt{e_{s} e_{t}} \text { with } e_{s}, e_{t}<0,
$$

in this limit, the parameter $|\rho| \rightarrow \infty$.

The only eigenvector of the matrix $\hat{Q}_{T=0}\left(\boldsymbol{\Delta}_{0}\right)$ with a non-vanishing eigenvalue corresponds to $\boldsymbol{\Delta}_{0}=\boldsymbol{\Delta}^{\prime}$, which is given by equation (32). Thus, the ratio between the spin-singlet and the spin-triplet components at $T=0$ is equal to that obtained for $T=T_{c}$, i.e. $\nu_{0}=\nu_{l}$. This result is a first indication that the form of the order parameter is unchanged upon temperature when the subdominant channel is inactive. This is confirmed by the numerical solution of equation (25).

Figure 3 shows the spin-singlet $\psi$ and the spin-triplet component $d$ as a function of temperature obtained for

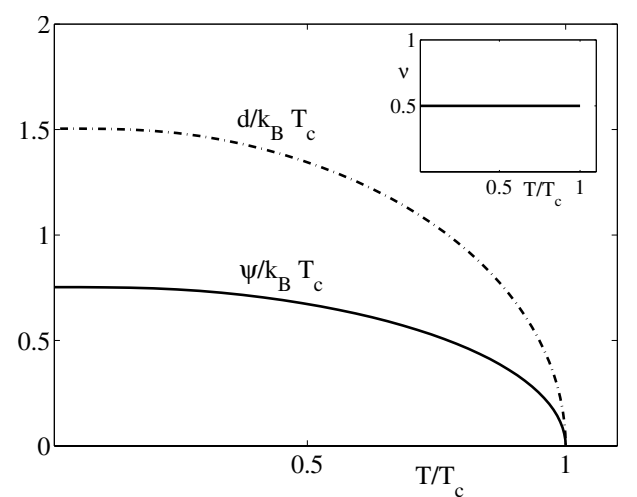

Fig. 3. The order parameter $\boldsymbol{\Delta}=(\psi, d)$ as a function of the temperature when the subdominant channel is inactive, i.e. $\lambda^{\prime \prime}=0$. The inset plot shows the ratio $\nu=\psi / d .\left(\nu_{l}=0.5\right.$, $\delta=-0.5)$.

$\nu=0.5$ and $\delta=-0.5$. The inset shows the ratio $\nu=\psi / d$. This does not depend on temperature. Furthermore, in this case there are explicit expressions for the two components

$$
\begin{aligned}
& \frac{d_{0} \gamma}{k_{B} T_{c} \pi}= \\
& \exp \left\{-\frac{\left\langle\left(1+\delta_{N}\right)\left(\nu+\left|\boldsymbol{g}_{\boldsymbol{k}}\right|\right)^{2} \ln \left(|\nu+| \boldsymbol{g}_{\boldsymbol{k}}||\right)\right\rangle}{\left\langle\left(1+\delta_{N}\right)\left(\nu+\left|\boldsymbol{g}_{\boldsymbol{k}}\right|\right)^{2}+\left(1-\delta_{N}\right)\left(\nu-\left|\boldsymbol{g}_{\boldsymbol{k}}\right|\right)^{2}\right\rangle}\right\} \\
& \times \exp \left\{-\frac{\left\langle\left(1-\delta_{N}\right)\left(\nu-\left|\boldsymbol{g}_{\boldsymbol{k}}\right|\right)^{2} \ln \left(|\nu-| \boldsymbol{g}_{\boldsymbol{k}}||\right)\right\rangle}{\left\langle\left(1+\delta_{N}\right)\left(\nu+\left|\boldsymbol{g}_{\boldsymbol{k}}\right|\right)^{2}+\left(1-\delta_{N}\right)\left(\nu-\left|\boldsymbol{g}_{\boldsymbol{k}}\right|\right)^{2}\right\rangle}\right\} \\
& \frac{\psi_{0} \gamma}{k_{B} T_{c} \pi}=\left\{\begin{array}{l}
\left\langle\left(1+\delta_{N}\right)\left(\nu+\left|\boldsymbol{g}_{\boldsymbol{k}}\right|\right)^{2} \ln \left(\left|1+\frac{\left|\boldsymbol{g}_{\boldsymbol{k}}\right|}{\nu}\right|\right)\right\rangle \\
\left.\exp \left\{-\frac{\left|\boldsymbol{g}_{\boldsymbol{k}}\right|}{\nu} \mid\right)\right\rangle
\end{array}\right. \\
& \quad \times \exp \left\{-\frac{\left\langle\left(1-\delta_{N}\right)\left(\nu-\left|\boldsymbol{g}_{\boldsymbol{k}}\right|\right)^{2} \ln \left(|1-| \boldsymbol{g}_{\boldsymbol{k}} \mid\right)^{2}+\left(1-\delta_{N}\right)\left(\nu-\left|\boldsymbol{g}_{\boldsymbol{k}}\right|\right)^{2}\right\rangle}{\left\langle\left(1+\delta_{N}\right)\left(\nu+\left|\boldsymbol{g}_{\boldsymbol{k}}\right|\right)^{2}+\left(1-\delta_{N}\right)\left(\nu-\left|\boldsymbol{g}_{\boldsymbol{k}}\right|\right)^{2}\right\rangle}\right\}
\end{aligned}
$$

of the order parameter. For a pure spin-singlet order parameter, $\nu \rightarrow \infty$, and for the pure protected spin-triplet state, $\nu=0$, we recover the standard result: $\psi_{0} / k_{B} T_{c}=$ $\pi / \gamma \approx 1.76$, and $d_{0} / k_{B} T_{c}=\pi / \gamma \exp \left\langle-\left|\boldsymbol{g}_{\boldsymbol{k}}\right|^{2} \ln \left(\left|\boldsymbol{g}_{\boldsymbol{k}}\right|\right)\right\rangle \approx 1.65$ for $\boldsymbol{g}_{\boldsymbol{k}} \propto\left(k_{y},-k_{x}, 0\right)$, respectively.

\section{The case $\lambda^{\prime \prime} \neq 0$}

However, if the subdominant channel is active, the ratio $\nu_{0} \equiv \psi_{0} / d_{0}$ between the spin-singlet and the spin-triplet 
components,

$$
\begin{aligned}
& \nu_{0}= \\
& -\frac{\left(Q_{3}^{0}-Q_{2}^{0} \delta\right)\left(1+\nu_{l}^{2}+2 \nu_{l} \delta\right)-\left(1-\delta^{2}\right)\left(Q_{3}^{0} \kappa+\nu_{l}\left(Q_{2}^{0} \kappa+\nu_{l}+\delta\right)\right) \rho}{\left(Q_{2}^{0}-Q_{1}^{0} \delta\right)\left(1+\nu_{l}^{2}+2 \nu_{l} \delta\right)-\left(Q_{2}^{0} \kappa-\nu_{l}+Q_{1}^{0} \kappa \nu_{l}-\delta\right)\left(1-\delta^{2}\right) \rho}
\end{aligned}
$$

at $T=0$ is in general different from that obtained for $T=T_{c}$. Moreover, $\nu_{0}$ depends on the cut-off energy $\epsilon_{c}$ via $\kappa$. The magnitude of the order parameter, which follows combining equation (45) with the equation

$$
\frac{\rho\left[\nu_{l}^{2} Q_{1}^{0}+Q_{3}^{0}+2 \nu_{l} Q_{2}^{0}\right]}{\nu_{l}^{2}+1+2 \nu_{l} \delta}=\frac{\left(Q_{1}^{0} Q_{3}^{0}-Q_{2}^{0^{2}}\right)}{\left(1-\delta^{2}\right)},
$$

which is obtained from the condition $\lambda_{i}^{0}\left(\boldsymbol{\Delta}_{i}^{0}\right)=1$, also depends on $\epsilon_{c}$. However, no changes in the qualitative behaviors of the gaps as a function of temperature have been observed solving equation (25) for different values of the cut-off energy $\epsilon_{c}$.

Comparing the condensation energy at $T=0$,

$$
\begin{aligned}
E_{c} & =-\frac{N_{1}}{4}\left\langle\left|\Delta_{1}(\boldsymbol{k})\right|^{2}\right\rangle_{\boldsymbol{k}}-\frac{N_{2}}{4}\left\langle\left|\Delta_{2}(\boldsymbol{k})\right|^{2}\right\rangle_{\boldsymbol{k}} \\
& =-\frac{N_{0}}{2}\left(\left|\psi_{0}\right|^{2}+\left|d_{0}\right|^{2}+2 \delta \psi_{0} d_{0}\right)
\end{aligned}
$$

obtained for different cases, we found that

$$
\left|E_{c}^{A}\right|>\left|E_{c}^{0}\right|>\left|E_{c}^{R}\right| \text {. }
$$

Here, we introduce $E_{c}^{R}$ and $E_{c}^{A}$ to denote the condensation energy obtained in the presence of a repulsive, $\lambda^{\prime \prime}<0$, and attractive, $\lambda^{\prime \prime}>0$, subdominant channel, respectively. $E_{c}^{0}$ denotes the limit case characterized by $\lambda^{\prime \prime}=0$. The Copper pair gains condensation energy if the subdominant channel is attractive. On the contrary, the condensation energy of the pairs decreases in the presence of a repulsive subdominant channel.

Figure 4 shows the order parameter as a function of the temperature obtained for two representative cases. The first case is characterised by a repulsive, $\lambda^{\prime \prime}<0$, subdominant channel with $\lambda^{\prime \prime} / \lambda^{\prime} \approx-5000$, while the second is characterized by an attractive, $\lambda^{\prime \prime}>0$, subdominant channel with $T_{c}^{\prime \prime} / T_{c}=0.7$. In both cases, $\nu=\psi / d=0.5$ and $\boldsymbol{g}_{\boldsymbol{k}} \propto\left(k_{y},-k_{x}, 0\right)$. This means that the nucleation of the superconducting state is characterized in both cases by two line nodes, perpendicular to the $z$-axis, on the gap amplitude, $\Delta_{2}(\theta)=(\psi-d \sqrt{3 / 2}|\sin (\theta)|)$, of the second non-degenerated band. The polar angle $\theta_{ \pm}$determines the position of those nodes. In our example, the two line nodes are characterised by $\theta_{ \pm}=\pi / 2 \pm(\pi / 2-\arcsin (\sqrt{2 / 3} \nu)$ ) (we suppose a native spherical Fermi surface). From the insets of Figure 4 we see that the ratio $\nu$ between the spin-singlet and the spin-triplet component now changes with the temperature. Thus the position of the nodes also now depends upon temperature. To the right of the graphs in Figure 4 we draw the gap structure on the second Fermi surface schematically. The arrows show the directions along which
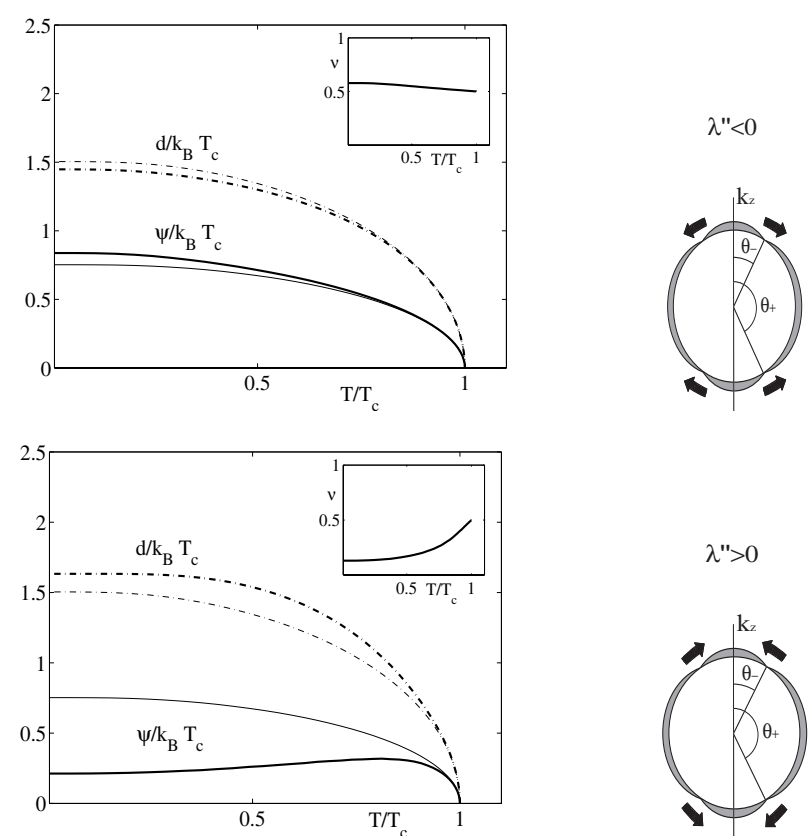

Fig. 4. The order parameter $\boldsymbol{\Delta}=(\psi, d)$ as a function of temperature. The first plot shows the case with a repulsive, $\lambda^{\prime \prime}<0$, subdominant channel, and $\lambda^{\prime \prime} / \lambda^{\prime} \approx-5000$. The second plot shows the result obtained when the subdominant channel is attractive, $\lambda^{\prime \prime}>0$, and characterized by $T_{c}^{\prime \prime} / T_{c}=0.7$. The thin lines are obtained for $\lambda^{\prime \prime}=0 .\left(\nu_{l}=\psi / d=0.5, \delta=-0.5\right.$, $\left.\epsilon_{c}=60 k_{B} T_{c}\right)$.

the accidental line nodes move, decreasing the temperature. This last depends on the character of the subdominant channel.

In the case of an attractive subdominant channel there is the possibility of a second phase transition. This is the case if there is a second solution $\Delta_{0}^{\prime \prime}=\left(\psi_{0}^{\prime \prime}, d_{0}^{\prime \prime}\right)$ for equations $(45,46)$ with

$$
\left|\psi_{0}^{\prime \prime}\right|^{2}+\left|d_{0}^{\prime \prime}\right|^{2}+2 \delta \psi_{0}^{\prime \prime} d_{0}^{\prime \prime}>\left|\psi_{0}\right|^{2}+\left|d_{0}\right|^{2}+2 \delta \psi_{0} d_{0}
$$

Our numerical investigation seems to show that in general there is no second phase transition. The solution nucleating at the highest critical temperature seems to maintain the highest condensation energy, changing its structure continuously with temperature. The only exception affects systems with constant density of state, $\delta=0$, and with no mixing pairing interaction component, $e_{m}=0$. In this particular case, we could have a second transition between a spin-triplet state and a spin-singlet state.

\section{The effect of the disorder}

Effects of disorder are described by potential scattering of the quasiparticles, which in real-space representation is given by

$$
\mathcal{H}_{\text {imp }}=\sum_{i} \mathcal{H}_{i}, \quad \mathcal{H}_{i}=\int u\left(\boldsymbol{r}-\boldsymbol{r}_{i}\right) \psi_{s}^{\dagger}(\boldsymbol{r}) \psi_{s}(\boldsymbol{r}) d \boldsymbol{r}
$$



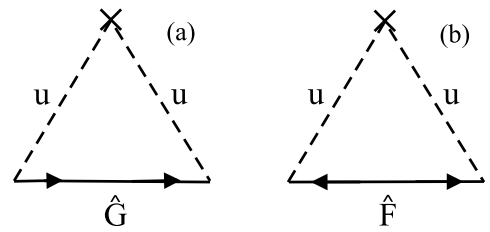

Fig. 5. The-self energy contributions due to impurity scattering in the Born approximation, of normal type $\Sigma_{G}$ (a) and of anomalous type $\Sigma_{F}$ (b).

where $u(\boldsymbol{r})$ is the potential of a non-magnetic impurity, which we consider rather short-ranged, such that $s$-wave scattering is dominant. We are interested in the disorder-averaged normal and anomalous Green's functions $\hat{G}$ and $\hat{F}$,

$$
\begin{aligned}
G_{\lambda \mu}\left(r-r^{\prime}\right) & =-\left\langle T_{\tau}\left\{\psi_{\lambda}(r) \psi_{\mu}^{\dagger}\left(r^{\prime}\right)\right\}\right\rangle \\
F_{\lambda \mu}\left(r-r^{\prime}\right) & =\left\langle T_{\tau}\left\{\psi_{\lambda}(r) \psi_{\mu}\left(r^{\prime}\right)\right\}\right\rangle \\
F_{\lambda \mu}^{\dagger}\left(r-r^{\prime}\right) & =\left\langle T_{\tau}\left\{\psi_{\lambda}^{\dagger}(r) \psi_{\mu}^{\dagger}\left(r^{\prime}\right)\right\}\right\rangle,
\end{aligned}
$$

with $r=(\boldsymbol{r}, \tau)$ and where the bracket denotes the thermal average.

For the impurity average, we use the Born approximation [22], neglecting the possibility of more than two scattering events at the same impurity, which is valid provided that the potential is small in comparison with the characteristic electron energy scale $\epsilon_{F}\left(\epsilon_{F}=\right.$ Fermi energy, or analogue the band width).

Formally, the impurity scattering enters the self-energy of the Greens function of the normal, $\hat{\Sigma}_{G}$, Figure 5 a, and the anomalous type, $\hat{\Sigma}_{F}$, see Figure 5 b. Their mathematical expressions read

$$
\begin{aligned}
& \hat{\Sigma}_{G}\left(i \omega_{n}\right)=\frac{\Gamma}{\pi N_{0}} \int \frac{d \boldsymbol{k}^{\prime}}{(2 \pi)^{3}} \hat{G}\left(\boldsymbol{k}^{\prime}, i \omega_{n}\right) \\
& \hat{\Sigma}_{F}\left(i \omega_{n}\right)=\frac{\Gamma}{\pi N_{0}} \int \frac{d \boldsymbol{k}^{\prime}}{(2 \pi)^{3}} \hat{F}\left(\boldsymbol{k}^{\prime}, i \omega_{n}\right),
\end{aligned}
$$

where $\Gamma \equiv \pi n_{i m p} N_{0} u^{2}$ is the averaged scattering rate, $N_{0} \equiv\left(N_{1}+N_{2}\right) / 2$ and $N_{1,2}$ are the densities of state (DOS) of the two bands at the Fermi level. In addition, we have introduced the impurity concentration $n_{i m p}$ and the $s$-wave scattering potential $u^{2}$.

The Gor'kov equations with these self-energy contributions are formally analogous to those obtained for systems with an inversion symmetry [22]

$$
\begin{gathered}
\left(\hat{G}_{0}^{-1}\left(\boldsymbol{k}, i \omega_{n}\right)-\hat{\Sigma}_{G}\left(i \omega_{n}\right)\right) \hat{G}\left(\boldsymbol{k}, i \omega_{n}\right) \\
+\left(\hat{\Delta}(\boldsymbol{k})+\hat{\Sigma}_{F}\left(i \omega_{n}\right)\right) \hat{F}^{\dagger}\left(\boldsymbol{k}, i \omega_{n}\right)=\hat{\sigma}_{0} \\
\left(\hat{G}_{0}^{-1^{\top}}\left(-\boldsymbol{k},-i \omega_{n}\right)+\hat{\Sigma}_{G}^{\top}\left(-i \omega_{n}\right)\right) \hat{F}^{\dagger}\left(\boldsymbol{k}, i \omega_{n}\right) \\
-\left(\hat{\Delta}^{\dagger}(\boldsymbol{k})+\hat{\Sigma}_{F}^{\dagger}\left(i \omega_{n}\right)\right) \hat{G}\left(\boldsymbol{k}, i \omega_{n}\right)=0 .
\end{gathered}
$$

The two-band Green's functions $G_{1,2}$ and $F_{1,2}$, are derived by unitary transformation (5), as already done for the clean system (16)

$$
\begin{gathered}
\left(\left\{G_{1,2}^{0}\left(\boldsymbol{k}, i \omega_{n}\right)\right\}^{-1}-\Sigma_{G}\left(i \omega_{n}\right)\right) G_{1,2}\left(\boldsymbol{k}, i \omega_{n}\right) \\
+\left(\Delta_{1,2}(\boldsymbol{k})+\Sigma_{F}\left(i \omega_{n}\right)\right) F_{1,2}^{\dagger}\left(\boldsymbol{k}, i \omega_{n}\right)=1 \\
\left(\left\{G_{1,2}^{0}\left(-\boldsymbol{k},-i \omega_{n}\right)\right\}^{-1}+\Sigma_{G}\left(-i \omega_{n}\right)\right) F_{1,2}^{\dagger}\left(\boldsymbol{k}, i \omega_{n}\right) \\
-\left(\Delta_{1,2}^{*}(\boldsymbol{k})+\Sigma_{F}\left(i \omega_{n}\right)\right) G_{1,2}\left(\boldsymbol{k}, i \omega_{n}\right)=0,
\end{gathered}
$$

where, in this case,

$$
\begin{aligned}
& \Sigma_{G}\left(i \omega_{n}\right)=\frac{\Gamma}{2 \pi N_{0}} \int \frac{d \boldsymbol{k}^{\prime}}{(2 \pi)^{3}}\left\{G_{1}\left(\boldsymbol{k}^{\prime}, i \omega_{n}\right)+G_{2}\left(\boldsymbol{k}^{\prime}, i \omega_{n}\right)\right\} \\
& \Sigma_{F}\left(i \omega_{n}\right)=\frac{\Gamma}{2 \pi N_{0}} \int \frac{d \boldsymbol{k}^{\prime}}{(2 \pi)^{3}}\left\{F_{1}\left(\boldsymbol{k}^{\prime}, i \omega_{n}\right)+F_{2}\left(\boldsymbol{k}^{\prime}, i \omega_{n}\right)\right\} .
\end{aligned}
$$

Thus the Gor'kov equations are still diagonal in the band index. The scattering on an impurity does not change the spin of a quasiparticle and with the impurity-average a certain translational symmetry is restored such that the two bands do not mix in this approximation. Interband effects occur only through virtual processes.

Introducing the modified gap functions $\tilde{\Delta}_{1,2}\left(\boldsymbol{k}, i \omega_{n}\right)=$ $\Delta_{1,2}(\boldsymbol{k})+\Sigma_{F}\left(i \omega_{n}\right)$ and frequencies $i \tilde{\omega}_{n}=i \omega_{n}-\Sigma_{G}\left(i \omega_{n}\right)$, the solution of the two-band Gor'kov equations is given by

$$
\begin{aligned}
& G_{1,2}\left(\boldsymbol{k}, i \omega_{n}\right)=-\frac{i \tilde{\omega}_{n}+\xi_{1,2}}{\left(\tilde{\omega}_{n}^{2}+\left|\tilde{\Delta}_{1,2}\right|^{2}+\xi_{1,2}^{2}\right)}, \\
& F_{1,2}\left(\boldsymbol{k}, i \omega_{n}\right)=\frac{\tilde{\Delta}_{1,2}}{\left(\tilde{\omega}_{n}^{2}+\left|\tilde{\Delta}_{1,2}\right|^{2}+\xi_{1,2}^{2}\right)},
\end{aligned}
$$

in which $\tilde{\Delta}_{1,2}\left(\boldsymbol{k}, i \omega_{n}\right)$ and $\tilde{\omega}_{n}$ have to be determined selfconsistently. The corresponding equations are given by the substitution of equations (56) and (57) into equations (22) and (55),

$$
\begin{aligned}
\tilde{\omega}_{n} & =\omega_{n}+\tilde{\omega}_{n} \Gamma Q_{1}\left(i \tilde{\omega}_{n}\right), \\
\tilde{\psi} & =\psi+\tilde{\psi} \Gamma Q_{1}\left(i \tilde{\omega}_{n}\right)+d \Gamma Q_{2}\left(i \tilde{\omega}_{n}\right), \\
\frac{1}{N_{0} V} \boldsymbol{\Delta} & =\pi k_{B} T \sum_{\omega_{n}>-\epsilon_{c}}^{\epsilon_{c}} \hat{Q}\left(i \tilde{\omega}_{n}\right) \Delta,
\end{aligned}
$$

where in the last equation $\boldsymbol{\Delta}=(\psi, d)$, and

$$
\begin{aligned}
\hat{Q}\left(i \tilde{\omega}_{n}\right)= & \frac{Q_{1}\left(i \tilde{\omega}_{n}\right)}{1-\Gamma Q_{1}\left(i \tilde{\omega}_{n}\right)}\left(\begin{array}{cc}
-e_{s} & 0 \\
e_{m} & 0
\end{array}\right) \\
& +\frac{Q_{2}\left(i \tilde{\omega}_{n}\right)}{1-\Gamma Q_{1}\left(i \tilde{\omega}_{n}\right)}\left(\begin{array}{cc}
e_{m} & -e_{s} \\
-e_{t} & e_{m}
\end{array}\right) \\
& +\left\{Q_{3}\left(i \tilde{\omega}_{n}\right)+\frac{\Gamma Q_{2}^{2}\left(i \tilde{\omega}_{n}\right)}{1-\Gamma Q_{1}\left(i \tilde{\omega}_{n}\right)}\right\}\left(\begin{array}{cc}
0 & e_{m} \\
0 & -e_{t}
\end{array}\right)
\end{aligned}
$$


is defined using the functions $Q_{1}, Q_{2}, Q_{3}$, which are obtained by substituting $\psi$ and $\omega_{n}$ by $\tilde{\psi}$ and $\tilde{\omega}_{n}$ in equation (27), respectively.

The effect of disorder on the superconducting instability is deduced from the solution of the linearized form of the self-consistent system of equations (58-60). Using standard summation techniques [18], we find

$$
\frac{1}{N_{0} V} \boldsymbol{\Delta}=\left\{f_{1}\left(\epsilon_{c}, k_{B} T\right) \hat{Q}^{l}+f_{2}\left(\Gamma, k_{B} T\right) \hat{Q}_{\Gamma}^{l}\right\} \boldsymbol{\Delta},
$$

where $f_{1}$ and $\hat{Q}^{l}$ have been defined in equations (29) and (30), respectively. Instead, $f_{2}$ is defined by

$$
f_{2}\left(\Gamma, k_{B} T\right)=\Psi\left(\frac{1}{2}+\frac{\Gamma}{2 \pi k_{B} T}\right)-\Psi\left(\frac{1}{2}\right)
$$

and

$$
\hat{Q}_{\Gamma}^{l}=\left(1-\delta_{N}^{2}\left\langle\left|\boldsymbol{g}_{\boldsymbol{k}}\right|\right\rangle^{2}\right)\left(\begin{array}{cc}
0 & -e_{m} \\
0 & e_{t}
\end{array}\right)
$$

Here we have used the digamma function $\Psi(z)$ defined by $\Psi(z) \equiv d / d z \ln (z !)$.

First, we would like to note that the zeros in the first column of the matrix $\hat{Q}_{\Gamma}^{l}$ are a consequence of Anderson's theorem [23], i.e. the conventional $s$-wave pairing state is not affected by non-magnetic impurities. For more general states in our two-dimensional order parameter space, $T_{c}$ decreases with growing disorder. We distinguish two basically different cases here: For the pure system either both eigenvalues $\lambda^{\prime}, \lambda^{\prime \prime}$ are positive, or one of the two, $\lambda^{\prime \prime}$ is negative. In the first case, there is a second lower (bare) critical temperature $k_{B} T_{c}^{\prime \prime}=1.14 \epsilon_{c} \exp \left(-1 /\left(N_{0} V \lambda^{\prime \prime}\right)\right)$.

\section{The case $\lambda^{\prime \prime}=0$}

Before starting the discussion of these two general cases, we consider the boundary situation with $\lambda^{\prime \prime}=0$. The only eigenvector of the matrix $\hat{Q}_{\Gamma}^{l}$ with a non-vanishing eigenvalue corresponds to the form of $\boldsymbol{\Delta}^{\prime}$ given by in equation (32). Hence, in this case, disorder would not alter the structure of the nucleating order parameter

$$
\boldsymbol{\Delta}=\left(\begin{array}{l}
\psi \\
d
\end{array}\right) \propto \boldsymbol{\Delta}^{\prime} \propto\left(\begin{array}{c}
\sqrt{\left|e_{s}\right|} \\
\operatorname{sign}\left(e_{m}\right) \sqrt{\left|e_{t}\right|}
\end{array}\right)
$$

The instability equation (62) becomes

$$
\frac{1}{N_{0} V}=\lambda^{\prime} f_{1}\left(\epsilon_{c}, k_{B} T\right)+e_{t}\left(1-\delta^{2}\right) f_{2}\left(\Gamma, k_{B} T\right),
$$

where $e_{t}\left(1-\delta^{2}\right)$ is the eigenvalue of the matrix $\hat{Q}_{\Gamma}^{l}$. We replace $f_{1}=\ln \left(T_{c} / T\right)+1 / N_{0} V \lambda^{\prime}$ and $\lambda^{\prime}=-\left(e_{s}+e_{t}\right)+$ $2 e_{m} \delta$ in equation (66) and find

$$
\ln \left(T_{c} / T\right)=\frac{e_{t}\left(1-\delta^{2}\right)}{\left(e_{s}+e_{t}\right)-2 e_{m} \delta} f_{2}\left(\Gamma, k_{B} T\right) .
$$

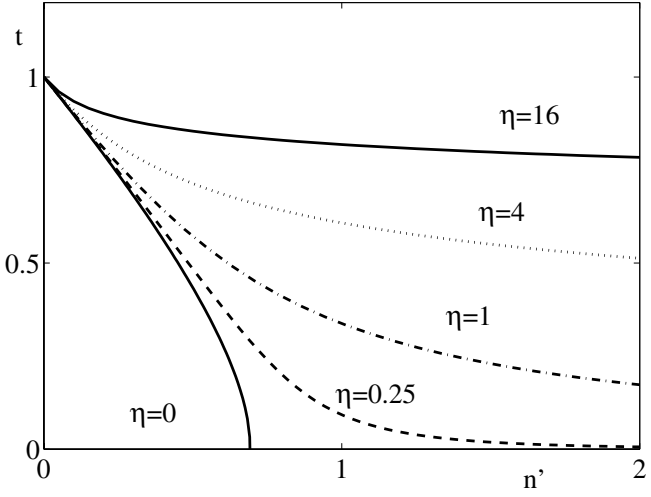

Fig. 6. The critical temperature as a function of the impurity concentration reduced so as to have a slope of -1 at $t=1$ for different values of $\eta$. For the value of $\eta$, see equation (69). This result is valid only for $\lambda^{\prime \prime}=0$.

Using equation (65) we obtain

$$
\ln \left(\frac{1}{t}\right)=\frac{1}{1+\eta} f_{2}(\gamma, t)
$$

where

$$
\eta=\frac{(\psi+\delta d)^{2}}{d^{2}\left(1-\delta^{2}\right)},
$$

with $t=T / T_{c}$ and $\gamma=\Gamma / k_{B} T_{c}$. The effect of impurity scattering affects $T_{c}$ as in unconventional superconductors in general [24]. However, a distinctive point is the presence of the pre-factor $1 /(1+\eta)$. A similar result was obtained for the $\mathrm{s}+\mathrm{g}$-superconductivity in borocarbides [25].

In order to visualize the behavior of the onset temperature of superconductivity depending on impurity concentrations, we introduce the normalization with respect to the initial slope of $T_{c}$-reduction,

$$
n^{\prime}=\frac{n_{i m p}}{\left(-d n_{i m p} / d t\right)_{t=1}}=\frac{\tilde{\gamma}}{(-d \tilde{\gamma} / d t)_{t=1}} .
$$

Figure 6 shows the evolution of $t$ as a function of $n^{\prime}$ for different values of $\eta$ when the subdominant channel has $\lambda^{\prime \prime}=0$

For $\eta>0$, the superconducting instability extends to large impurity concentrations $n^{\prime}$ with the asymptotical behavior of the critical temperature

$$
0.88 t^{-\eta}-t=\frac{4(1+\eta)}{\pi} n^{\prime}
$$

Hence, we observe a variation of the robustness against non-magnetic impurities. For $\eta=0$, the standard behavior of an unconventional superconductor is found, while for $\eta \rightarrow \infty$ the non-sensitivity against disorder analogous to a conventional superconducting phase is realized. The latter case coincides with the suppression of the spin-triplet component in the pure phase. The exponent $\eta$ can be interpreted as the product of two ratios

$$
\eta=\eta_{1} \eta_{2} \text { with } \eta_{1,2}=\frac{\left\langle N_{1} \Delta_{1}(\boldsymbol{k})+N_{2} \Delta_{2}(\boldsymbol{k})\right\rangle}{N_{1,2}\left\langle\Delta_{1}(\boldsymbol{k})-\Delta_{2}(\boldsymbol{k})\right\rangle}
$$


where $\eta_{1,2}$ corresponds to the ratio between the $s$-wave spin-singlet component (numerator) and the spin-triplet component lying in the first and second band (denominator), respectively. Once $\left\langle N_{1} \Delta_{1}(\boldsymbol{k})+N_{2} \Delta_{2}(\boldsymbol{k})\right\rangle \neq 0$, the robustness of the superconducting state against disorder is introduced by the presence of a $s$-wave spin-singlet contribution.

For other unconventional pairing states which do not belong to the trivial representation $A_{1}$, the impurity contribution to the self-energy of anomalous type is zero and we recover exactly the result obtained by Larkin [24].

\section{The case $\lambda^{\prime \prime} \neq 0$}

In this case, the eigenvectors of the two matrices $\hat{Q}^{l}$ and $\hat{Q}_{\Gamma}^{l}$ are different, such that we need to diagonalize the matrix appearing in equation (62). The highest eigenvalue and its eigenvector read

$$
\begin{aligned}
& 2 \lambda=2 \lambda^{\prime} f_{1}+c_{1}-c_{2}+\sqrt{c_{2}^{2}+c_{3}}, \\
& \Delta \propto\left(\begin{array}{c}
\psi^{\prime}+\frac{\sqrt{c_{2}^{2}+c_{3}}-c_{4}}{f_{1} c_{5}} \\
d^{\prime}
\end{array}\right),
\end{aligned}
$$

where $\left(\psi^{\prime}, d^{\prime}\right)$ are normalized $\left(\psi^{\prime 2}+d^{\prime 2}=1\right)$. Furthermore, the parameters $c_{i}$ are defined as

$$
\begin{aligned}
& c_{1}=\left(1-\delta^{2}\right)\left[e_{t}-\frac{e_{t}\left(e_{t}-e_{s}-2 e_{m} \delta\right)+2 e_{m}^{2}}{\Lambda}\right] f_{2}, \\
& c_{2}=\Lambda f_{1}-\frac{\left(1-\delta^{2}\right)}{2}\left\{\Lambda+\frac{e_{t}^{2}+e_{s}\left[4 \delta\left(e_{m}-e_{t} \delta\right)-e_{s}\right]}{\Lambda}\right\} f_{2}, \\
& c_{3}=-\frac{4\left(e_{m}^{2}-e_{s} e_{t}\right)\left(e_{m}-e_{t} \delta\right)^{2}\left(\delta^{2}-1\right)^{2}}{\Lambda^{2}} f_{2}^{2}, \\
& c_{4}=\Lambda f_{1}+e_{t}\left(1-\delta^{2}\right) f_{2}, \\
& c_{5}=\sqrt{4\left(e_{m}-e_{t} \delta\right)^{2}+\left(e_{t}-e_{s}+\Lambda\right)^{2}} .
\end{aligned}
$$

The equation determining the critical temperature can be expressed as

$$
\frac{2}{N_{0} V}=2 \lambda^{\prime} f_{1}\left(\epsilon_{c}, k_{B} T\right)+c_{1}-c_{2}+\sqrt{c_{2}^{2}+c_{3}} .
$$

When we again use the relation $f_{1}=\ln \left(T_{c} / T\right)+1 / N_{0} V \lambda^{\prime}$, we can cancel the $2 / N_{0} V$ term appearing on the left-hand side of equation (76) and reach a new convenient representation,

$$
\left[2 \lambda^{\prime} \ln \left(\frac{1}{t}\right)+c_{1}\right]^{2}-2\left[2 \lambda^{\prime} \ln \left(\frac{1}{t}\right)+c_{1}\right] c_{2}-c_{3}=0 .
$$

We assume now that the second channel is attractive, $\lambda^{\prime \prime}>$ 0 , so that the clean system is characterized by a second lower (bare) critical temperature $t^{\prime \prime}=T_{c}^{\prime \prime} / T_{c}$. We use the

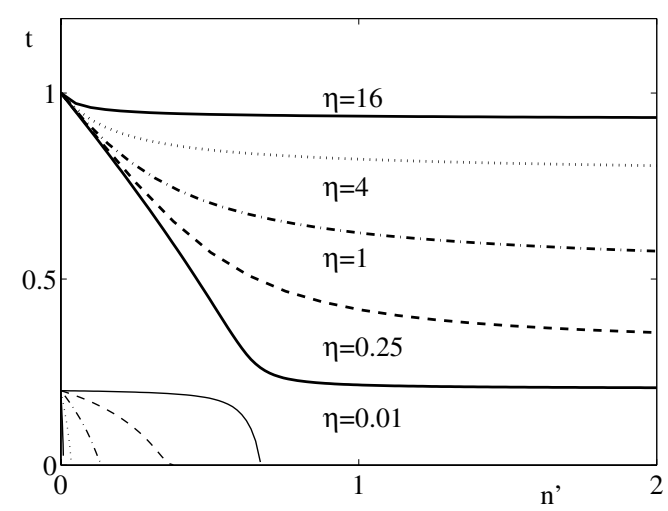

Fig. 7. The critical temperature as a function of the impurity concentration and for different values of $\eta$. Those corresponding to the dominant channel, thick lines, have been reduced so as to have a slope of -1 at $t=1$. The thin lines show the evolution of the subdominant channel. This result is obtained supposing that the subdominant channel is attractive and $t^{\prime \prime}=0.2$.

relation $f_{1}=\ln \left(T_{c} / T\right)+\ln \left(t^{\prime \prime}\right) \lambda^{\prime \prime} /\left(\lambda^{\prime \prime}-\lambda^{\prime}\right)$ to simplify equation (77). This leads to an equation of the second order in $\ln (t)$ of the following form which allows us to determine both transition temperatures $\left(t=T / T_{c}\right.$ or $t=$ $\left.T^{\prime \prime} / T_{c}\right)$

$$
\ln \left(\frac{1}{t}\right)\left(\ln \left(t^{\prime \prime}\right)-f_{2}(\gamma, t)-\ln (t)\right)=\frac{1}{1+\eta} f_{2}(\gamma, t) \ln \left(t^{\prime \prime}\right)
$$

where $\eta$ is now given by the form of the order parameter in the clean system

$$
\eta=\frac{\left(\psi^{\prime}+\delta d^{\prime}\right)^{2}}{d^{\prime 2}\left(1-\delta^{2}\right)}
$$

and $\left(\psi^{\prime}, d^{\prime}\right)$ are connected to the pairing potential via equation (32).

Figure 7 shows the solutions of equation (78) for different values of $\eta$ and for $t_{0}^{\prime \prime}=0.2$ in the pure case. The thick line shows the onset temperature of superconductivity as a function of $n^{\prime}$. The thin line correspond to the bare transition temperature of subdominant instability, as obtained from the square root appearing in equation (76).

The presence of an attractive subdominant channel supports the survival of superconductivity under nonmagnetic impurity scattering. The lowest limit for the critical temperature is given by

$$
\lim _{n^{\prime} \rightarrow \infty} t=t_{0}^{\prime \prime \frac{1}{1+\eta}} .
$$

Using equation (76) to simplify equation (74), we find that the form of the order parameter nucleating at the critical temperature $t$ is given by

$$
\left(\begin{array}{l}
\psi \\
d
\end{array}\right) \propto\left(\begin{array}{cc}
1+f_{3}(t) & \delta f_{3}(t) \\
0 & 1
\end{array}\right)\left(\begin{array}{l}
\psi^{\prime} \\
d^{\prime}
\end{array}\right),
$$

where we have introduced the function $f_{3}(t)$ defined as

$$
f_{3}(t)=-\frac{(1+\eta) \ln (t)}{(1+\eta) \ln (t)-\ln \left(t^{\prime \prime}\right)} .
$$




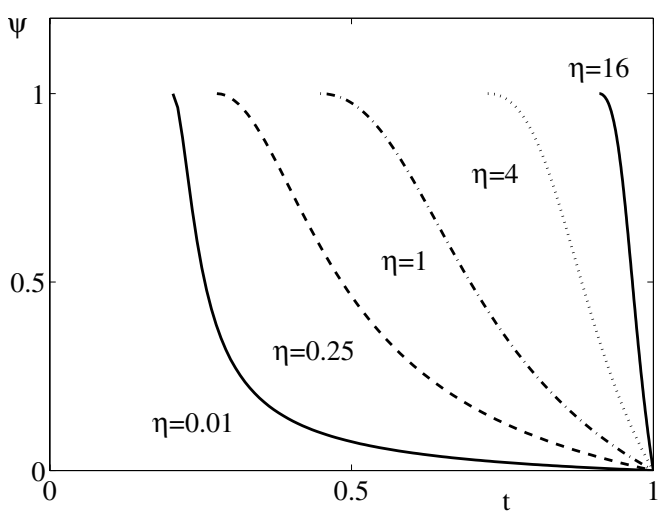

Fig. 8. The spin-singlet component $\psi$ of the normalized order parameter as a function of the critical temperature $t$ and for different values of $\eta$. This result is obtained supposing that the subdominant channel is attractive with $t^{\prime \prime}=0.2$ and that the order parameter of the clean system is spin-triplet .

The spin-singlet component of the order parameter increases with disorder. In the limit of a dirty system, the character of the order parameter is purely spin-singlet. Note that the subdominant instability is, in any case, suppressed by the disorder. The larger $\eta$, the stronger the suppression.

The behavior of the spin-singlet component $\psi$ of the normalized order parameter as a function of the critical temperature $t$ and for different values of $\eta$ is shown in Figure 8. The order-parameter of the clean system is supposed to be in the spin-triplet channel.

The case with a repulsive subdominant channel, $\lambda^{\prime \prime}<$ 0 , can be easily treated within equations (78) and (82), substituting $\ln \left(t^{\prime \prime}\right)$ with $1 / N_{0} V_{\text {eff }}$, equation (42). From equation (78) we find that superconductivity disappears for sufficiently high impurity concentrations. The critical impurity concentration $n_{c}^{\prime}$ is given by

$$
0.88 e^{\frac{1}{\Gamma_{0} V_{e f f}} \frac{\eta}{1+\eta}}=\frac{4(1+\eta)}{\pi} n_{c}^{\prime}
$$

Furthermore, the spin-singlet component of the nucleating order parameter decreases with disorder evolving towards

$$
\left(\begin{array}{l}
\psi_{c} \\
d_{c}
\end{array}\right) \propto\left(\begin{array}{c}
-\delta d^{\prime} \\
d^{\prime}
\end{array}\right)
$$

at the critical impurity concentration $n_{c}^{\prime}$.

\section{Conclusion}

In this paper we characterized the superconducting state belonging to the trivial representation $A_{1}$ in a material without an inversion center and strong antisymmetric spin-orbit coupling. The corresponding pairing state involves spin-singlet $s$-wave pairing as well as a spin-triplet component specific to the spin-orbit coupling. In addition to the pure spin-singlet $s$-wave and spin-triplet pairing, the pairing interaction includes a parity mixing contribution corresponding to an inter-parity scattering of Cooper pairs.

The combination of the three types of pairing interaction $\left(e_{s}, e_{t}, e_{m}\right)$ and the distribution of the density of states on the two non-degenerated bands $\delta$ determine the form of the order parameter represented by a singlet and a triplet component $\Delta=(\psi, d)$. The two-component structure of the order parameter allows for two distinct pairing channels, a dominant and a subdominant one. The pairing instability of the clean system is completely characterized by the property of the dominant channel. For the case of $\mathrm{CePt}_{3} \mathrm{Si}$, we found that if $|d|>|\psi|$ and $|\psi| \neq 0$, then one Fermi surface would have accidental line nodes perpendicular to the $z$-axis in the quasiparticle gap. This is the case if the spin-triplet pairing interaction is more attractive than the spin-singlet interaction, i.e. $e_{t}<0$ with $e_{t}<e_{s}$ and if $e_{m} \neq e_{s} \delta$. There is important experimental evidence confirming the realization of this superconducting state in $\mathrm{CePt}_{3} \mathrm{Si}$. In particular, the analysis of the results concerning the nuclear magnetic relaxation rate $1 / T_{1} T$, obtained by Pt-NMR [26-28] shows two behaviors which contradict the conventional picture of superconductivity. The NMR relaxation rate shows the Hebel-Slichter coherence peak, indicating an $s$-wave-like pairing, and at low temperature shows a power law, indicating unconventional pairing. Recent numerical calculations of $1 / T_{1} T$ [29] show that this situation is compatible with the realization of the "s-wave" state which we propose.

Decreasing the temperature the structure of the order parameter is strongly affected by the presence of the $s u b$ dominant channel. The condensation energy at $T=0$ of the superconducting state increases in the case of an attractive subdominant channel. The displacement direction of the line nodes as a function of the temperature will indicate if the subdominant channel is attractive or repulsive. The angle-resolved thermal conductivity and Josephson effect could give information on the character of the subdominant pairing channel. This methods are sensitive to the position of the line nodes. A possible movement of the line nodes with temperature should be detected.

The presence of two instabilities in the linearized gap equation, the dominant and subdominant one, provide the possibility that two superconducting phase transitions might appear. From our study, this possibility seems to be reduced to an exception.

We have also shown that depending on the properties characterizing the two channels, the $A_{1}$-phase is affected by non-magnetic impurities in different ways. In all cases the dependence of $T_{c}$ on the impurity concentration is very characteristic and could be used to establish the realization of the $A_{1}$-phase and the property of the subdominant pairing state.

The authors thank N. Hayashi, A. Koga, T.M. Rice, K. Wakabayashi, and Y. Yanase for useful discussions. This work was supported by the Swiss National Science Foundation and the NCCR MaNEP. D.F.A. was also supported the National Science Foundation Grant No. DMR-0318665. 


\section{Appendix A: Origin of mixed singlet-triplet pairing interaction $\mathbf{e}_{m}$}

In this Appendix, we consider a microscopic origin for the mixed singlet-triplet pairing interaction $e_{m}$ given in equation (13). In particular, we show that that the Dzyaloshinskii-Moriya [19,20] (DM) magnetic interaction gives a contribution to $e_{m}$. Such an interaction is well known to exist for magnetic systems that break inversion symmetry. We then show how this interaction can arise from a single particle Hamiltonian with the same structure as that given in equation (2)

$$
\mathcal{H}_{0}^{m}=\sum_{\boldsymbol{k}, s, s^{\prime}}\left[\xi_{\boldsymbol{k}}^{m} \sigma_{0}+\alpha^{m} \boldsymbol{g}_{\boldsymbol{k}} \cdot \boldsymbol{\sigma}\right]_{s s^{\prime}} c_{\boldsymbol{k}_{s}}^{\dagger} c_{\boldsymbol{k} s^{\prime}},
$$

for both a weakly interacting Fermi liquid and for a Hubbard model near half filling. However, the parameter of this microscopical Hamiltonian equation (85) should not be confused with those characterizing the phenomenological Hamiltonian equation (2). The microscopic and the effective Hamiltonian can be connected by a renormalisation group treatment. This kind of connection is strongly dependent on the shape of the Fermi surface and will not be discussed here. However, the symmetry properties of the Hamiltonian are not affected by this process. Thus, our analysis suffices to show that $e_{m}$ is not zero in general and may be in some cases provide a substantial contribution to the superconducting condensation energy.

\section{A.1 DM Interaction}

The DM interaction can be written

$$
H_{D M}=\frac{1}{N} \sum_{\boldsymbol{q}} i \boldsymbol{D}(\boldsymbol{q}) \cdot \boldsymbol{S}_{\boldsymbol{q}} \times \boldsymbol{S}_{-\boldsymbol{q}}
$$

where $\boldsymbol{D}(\boldsymbol{q})$ is a real vector that satisfies $\boldsymbol{D}(\boldsymbol{q})=$ $-\boldsymbol{D}(-\boldsymbol{q})$. Invariance of $H_{D M}$ under point group operations leads to the constraint $\tilde{R} \boldsymbol{D}_{R \boldsymbol{q}}=\boldsymbol{D}_{\boldsymbol{q}}$, where $\tilde{R}$ is the proper part of the rotation $(\tilde{R}=\operatorname{Det}(R) \times R)$. Note that $\boldsymbol{g}(\boldsymbol{k})$ satisfies the same symmetry relation $\left(\tilde{R} \boldsymbol{g}_{R \boldsymbol{k}}=\boldsymbol{g}_{\boldsymbol{k}}\right)$. Consequently, the two vectors $\boldsymbol{D}(\boldsymbol{q})$ and $\boldsymbol{g}_{\boldsymbol{q}}$ are not orthogonal. Extracting the pairing contribution from equation (86) leads to the following mixed singlet-triplet pairing interaction

$$
\left[-\boldsymbol{D}\left(\boldsymbol{k}-\boldsymbol{k}^{\prime}\right) \cdot \hat{\boldsymbol{\tau}}\right]_{s_{1} s_{2}} \hat{\tau}_{s_{2}^{\prime} s_{1}^{\prime}}^{\dagger}+\hat{\tau}_{s_{1} s_{2}}\left[\boldsymbol{D}\left(\boldsymbol{k}-\boldsymbol{k}^{\prime}\right) \cdot \hat{\boldsymbol{\tau}}\right]_{s_{2}^{\prime} s_{1}^{\prime}}^{\dagger} \cdot
$$

If we assume the form $\boldsymbol{D}\left(\boldsymbol{k}-\boldsymbol{k}^{\prime}\right)=e_{m}^{m}\left(\boldsymbol{g}_{\boldsymbol{k}^{\prime}}-\boldsymbol{g}_{\boldsymbol{k}}\right)$ and impose symmetry constraints that arise from Pauli exclusion, we obtain a term of the same form as the mixed parity term of equation (14). As will be shown below, this form for $\boldsymbol{D}$ can be justified.

\section{A.2 DM Interaction in a weakly interacting Fermi liquid}

The DM interaction is known to exist in materials without inversion symmetry. This implies that the DM interaction should arise as a consequence of the existence of $\boldsymbol{g}_{\boldsymbol{k}}$ in the single particle Hamiltonian. Here we calculate the contribution of $\boldsymbol{g}_{\boldsymbol{k}}$ to the DM interaction through a calculation of the spin susceptibility. In the normal state the static spin-susceptibility is given by

$$
\left.\chi_{i j}(\boldsymbol{q})=-\mu_{B}^{2} k_{B} T \sum_{\boldsymbol{k}} \sum_{\omega_{n}} \operatorname{tr}\left\{\hat{\sigma}_{i} \hat{G}\left(\boldsymbol{k}, \omega_{n}\right) \hat{\sigma}_{j} \hat{G}\left(\boldsymbol{k}+\boldsymbol{q}, \omega_{n}\right)\right)\right\} .
$$

Upon using the form for the normal state Green's functions and carrying out the trace over the spins it can be shown

$$
\begin{aligned}
\chi_{i j} & (\boldsymbol{q})=-2 \mu_{B}^{2} k_{B} T \sum_{\boldsymbol{k}} \sum_{\omega_{n}}\left\{\left[\delta_{i j}\left(1-\hat{\boldsymbol{g}}_{\boldsymbol{k}} \cdot \hat{\boldsymbol{g}}_{\boldsymbol{k}+\boldsymbol{q}}\right)\right.\right. \\
& +\left(\hat{\boldsymbol{e}}_{i} \cdot \hat{\boldsymbol{g}}_{\boldsymbol{k}}\right)\left(\hat{\boldsymbol{e}}_{j} \cdot \hat{\boldsymbol{g}}_{\boldsymbol{k}+\boldsymbol{q}}\right)+\left(\hat{\boldsymbol{e}}_{i} \cdot \hat{\boldsymbol{g}}_{\boldsymbol{k}+\boldsymbol{q}}\right)\left(\hat{\boldsymbol{e}}_{j} \cdot \hat{\boldsymbol{g}}_{\boldsymbol{k}}\right) \\
& \left.+i\left(\hat{\boldsymbol{e}}_{i} \times \hat{\boldsymbol{e}}_{j}\right) \cdot\left(\hat{\boldsymbol{g}}_{\boldsymbol{k}+\boldsymbol{q}}-\hat{\boldsymbol{g}}_{\boldsymbol{k}}\right)\right] G_{1}\left(\boldsymbol{k}, \omega_{n}\right) G_{1}\left(\boldsymbol{k}+\boldsymbol{q}, \omega_{n}\right) \\
& +\left[\delta_{i j}\left(1-\hat{\boldsymbol{g}}_{\boldsymbol{k}} \cdot \hat{\boldsymbol{g}}_{\boldsymbol{k}+\boldsymbol{q}}\right)+\left(\hat{\boldsymbol{e}}_{i} \cdot \hat{\boldsymbol{g}}_{\boldsymbol{k}}\right)\left(\hat{\boldsymbol{e}}_{j} \cdot \hat{\boldsymbol{g}}_{\boldsymbol{k}+\boldsymbol{q}}\right)\right. \\
& \left.+\left(\hat{\boldsymbol{e}}_{i} \cdot \hat{\boldsymbol{g}}_{\boldsymbol{k}+\boldsymbol{q}}\right)\left(\hat{\boldsymbol{e}}_{j} \cdot \hat{\boldsymbol{g}}_{\boldsymbol{k}}\right)-i\left(\hat{\boldsymbol{e}}_{i} \times \hat{\boldsymbol{e}}_{j}\right) \cdot\left(\hat{\boldsymbol{g}}_{\boldsymbol{k}+\boldsymbol{q}}-\hat{\boldsymbol{g}}_{\boldsymbol{k}}\right)\right] \\
& +G_{2}\left(\boldsymbol{k}, \omega_{n}\right) G_{2}\left(\boldsymbol{k}+\boldsymbol{q}, \omega_{n}\right) \\
& +\left[\delta_{i j}\left(1+\hat{\boldsymbol{g}}_{\boldsymbol{k}} \cdot \hat{\boldsymbol{g}}_{\boldsymbol{k}+\boldsymbol{q}}\right)-\left(\hat{\boldsymbol{e}}_{i} \cdot \hat{\boldsymbol{g}}_{\boldsymbol{k}}\right)\left(\hat{\boldsymbol{e}}_{j} \cdot \hat{\boldsymbol{g}}_{\boldsymbol{k}+\boldsymbol{q}}\right)\right. \\
& \left.-\left(\hat{\boldsymbol{e}}_{i} \cdot \hat{\boldsymbol{g}}_{\boldsymbol{k}+\boldsymbol{q}}\right)\left(\hat{\boldsymbol{e}}_{j} \cdot \hat{\boldsymbol{g}}_{\boldsymbol{k}}\right)-i\left(\hat{\boldsymbol{e}}_{i} \times \hat{\boldsymbol{e}}_{j}\right) \cdot\left(\hat{\boldsymbol{g}}_{\boldsymbol{k}+\boldsymbol{q}}+\hat{\boldsymbol{g}}_{\boldsymbol{k}}\right)\right] \\
& +G_{1}\left(\boldsymbol{k}, \omega_{n}\right) G_{2}\left(\boldsymbol{k}+\boldsymbol{q}, \omega_{n}\right) \\
& +\left[\delta_{i j}\left(1+\hat{\boldsymbol{g}}_{\boldsymbol{k}} \cdot \hat{\boldsymbol{g}}_{\boldsymbol{k}+\boldsymbol{q}}\right)-\left(\hat{\boldsymbol{e}}_{i} \cdot \hat{\boldsymbol{g}}_{\boldsymbol{k}}\right)\left(\hat{\boldsymbol{e}}_{j} \cdot \hat{\boldsymbol{g}}_{\boldsymbol{k}+\boldsymbol{q}}\right)\right. \\
& \left.-\left(\hat{\boldsymbol{e}}_{i} \cdot \hat{\boldsymbol{g}}_{\boldsymbol{k}+\boldsymbol{q}}\right)\left(\hat{\boldsymbol{e}}_{j} \cdot \hat{\boldsymbol{g}}_{\boldsymbol{k}}\right)+i\left(\hat{\boldsymbol{e}}_{i} \times \hat{\boldsymbol{e}}_{j}\right) \cdot\left(\hat{\boldsymbol{g}}_{\boldsymbol{k}+\boldsymbol{q}}+\hat{\boldsymbol{g}}_{\boldsymbol{k}}\right)\right] \\
+ & \left.G_{2}\left(\boldsymbol{k}, \omega_{n}\right) G_{1}\left(\boldsymbol{k}+\boldsymbol{q}, \omega_{n}\right)\right\} \cdot
\end{aligned}
$$

The DM interaction is antisymmetric under interchange of $i$ and $j$ :

$$
\begin{aligned}
\boldsymbol{D}(\boldsymbol{q})= & -2 \mu_{B}^{2} k_{B} T \sum_{\boldsymbol{k}, \omega_{n}}\left\{\left[G_{1}\left(\boldsymbol{k}, \omega_{n}\right) G_{1}\left(\boldsymbol{k}+\boldsymbol{q}, \omega_{n}\right)\right.\right. \\
& \left.-G_{2}\left(\boldsymbol{k}, \omega_{n}\right) G_{2}\left(\boldsymbol{k}+\boldsymbol{q}, \omega_{n}\right)\right]\left[\hat{\boldsymbol{g}}_{\boldsymbol{k}+\boldsymbol{q}}-\hat{\boldsymbol{g}}_{\boldsymbol{k}}\right] \\
& +\left[G_{2}\left(\boldsymbol{k}, \omega_{n}\right) G_{1}\left(\boldsymbol{k}+\boldsymbol{q}, \omega_{n}\right)\right. \\
& \left.\left.-G_{1}\left(\boldsymbol{k}, \omega_{n}\right) G_{2}\left(\boldsymbol{k}+\boldsymbol{q}, \omega_{n}\right)\right]\left[\hat{\boldsymbol{g}}_{\boldsymbol{k}+\boldsymbol{q}}+\hat{\boldsymbol{g}}_{\boldsymbol{k}}\right]\right\} .
\end{aligned}
$$

Carrying out the sum over Matsubara frequencies, expanding the expression for $\boldsymbol{D}(\boldsymbol{q})$ to linear order in $\boldsymbol{q}$ one 
finds

$$
\begin{aligned}
\boldsymbol{D}(\boldsymbol{q})= & 2 \mu_{B}^{2} \sum_{\boldsymbol{k}}\left\{\left[\frac{\nabla n_{2} \cdot \boldsymbol{q}}{\boldsymbol{\nabla} \xi_{2} \cdot \boldsymbol{q}}-\frac{\boldsymbol{\nabla} n_{1} \cdot \boldsymbol{q}}{\boldsymbol{\nabla} \xi_{1} \cdot \boldsymbol{q}}\right] \boldsymbol{q} \cdot \boldsymbol{\nabla} \hat{\boldsymbol{g}}\right. \\
& \left.+\hat{\boldsymbol{g}} \boldsymbol{q} \cdot\left[\frac{n_{1}-n_{2}}{\left(\xi_{1}-\xi_{2}\right)^{2}}\left(\boldsymbol{\nabla} \xi_{1}+\nabla \xi_{2}\right)-\frac{\boldsymbol{\nabla} n_{1}+\nabla n_{2}}{\xi_{1}-\xi_{2}}\right]\right\}
\end{aligned}
$$

where $\xi_{i}=\xi_{i}(\boldsymbol{k}), n_{i}=\left(e^{\beta \xi_{i}}+1\right)^{-1}$ is the Fermi distribution function for band $i$, and $\boldsymbol{\nabla}=\boldsymbol{\nabla}_{\boldsymbol{k}}$. To linear order in $\alpha$, this gives

$$
\boldsymbol{D}(\boldsymbol{q})=-\frac{8 \mu_{B}^{2} \alpha^{m}}{3} \sum_{\boldsymbol{k}} \frac{d^{2} n\left(\epsilon_{\boldsymbol{k}}^{m}\right)}{d \epsilon_{\boldsymbol{k}}^{22}} \boldsymbol{q} \cdot \nabla_{\boldsymbol{k}} \boldsymbol{g}_{\boldsymbol{k}}
$$

For example if $\boldsymbol{g}_{\boldsymbol{k}}=\boldsymbol{k} / k_{F}$ (valid for a material with point group $O$ ), this gives

$$
\boldsymbol{D}(\boldsymbol{q})=-\frac{8 \mu_{B}^{2} \alpha^{m}}{3} N_{0}^{\prime m} \frac{\boldsymbol{q}}{k_{F}}=-\frac{4}{3} \chi_{P}^{m} \frac{\alpha N_{0}^{\prime m}}{N_{0}^{m}} \frac{\boldsymbol{q}}{k_{F}}
$$

where $N_{0}^{\prime m}=\left.\frac{d N_{0}^{m}}{d \xi_{F}}\right|_{\xi=0}$ is the derivative of the density of states evaluated at the Fermi surface and $\chi_{P}$ the Pauli susceptibility. This is a perturbative form and the derivative of density of states is considered at the Fermi surface in the absence of spin-orbit coupling. Estimates for $\alpha^{m}$ show that the value can be a considerable fraction of the band width. This result shows that there is a second order perturbation contribution to the mixing term $e_{m}$ if the derivative in the density of state $N_{0}^{\prime m}$ is different from zero.

\section{A.3 DM interaction within the Hubbard for finite ASOC}

Here we derive the DM interaction in the strong coupling regime by finding the effective Hamiltonian that governs the low-energy excitations of the Hubbard model with ASOC in the large $U / t$ regime. The technique used to extract the Hamiltonian for the low-energy excitations is similar to that used for the derivation of the t-J model starting from the Hubbard model.

We choose as zeroth-order Hamiltonian the on site Coulomb repulsion

$$
\mathcal{U}=U \sum_{i} n_{i \uparrow} n_{i \downarrow}
$$

The eigenstates of $\mathcal{U}$ are Fock states in the Wannier representation. $\mathcal{U}$ divides the Fock space into two sub spaces:

$$
\begin{aligned}
S & =\left[\left|n_{1 \uparrow}, n_{1 \downarrow}, n_{2 \uparrow}, \cdots\right\rangle: \forall n_{i \downarrow}+n_{i \uparrow} \leq 1\right] \\
D & =\left[\left|n_{1 \uparrow}, n_{1 \downarrow}, n_{2 \uparrow}, \cdots\right\rangle: \exists n_{i \downarrow}+n_{i \uparrow}=2\right] .
\end{aligned}
$$

$D$ contain at least one doubly occupied site, and $S$ are all configurations with either one or zero electrons per site. The hopping

$$
\mathcal{T}=\mathcal{T}^{s}+\mathcal{T}^{a}
$$

where

$$
\begin{aligned}
\mathcal{T}^{s} & =-\sum_{i j, s s^{\prime}} t_{i j}^{m} \sigma_{0} c_{i s}^{\dagger} c_{j s^{\prime}} \\
\mathcal{T}^{a} & =-\sum_{i j, s s^{\prime}} i \boldsymbol{\alpha}_{i j}^{m} \cdot \boldsymbol{\sigma}_{s s^{\prime}} c_{i s}^{\dagger} c_{j s^{\prime}}
\end{aligned}
$$

contains now an antisymmetric term $\left(\boldsymbol{\alpha}_{i j}=-\boldsymbol{\alpha}_{j i}\right)$ corresponding to the ASOC contribution. This means that also the effective interaction resulting by the superexchange process has an antisymmetric part. This last correspond to the DM interaction

$$
\begin{aligned}
\mathcal{H}^{D M}= & -\sum_{i j, s_{1} \cdots s_{4}} i \frac{t_{i j}^{m} \boldsymbol{\alpha}_{i j}^{m} \cdot\left(\boldsymbol{\sigma}_{s_{1} s_{2}} \delta_{s_{3} s_{4}}-\boldsymbol{\sigma}_{s_{3} s_{4}} \delta_{s_{1} s_{2}}\right)}{U} \\
& \times c_{i s_{1}}^{\dagger} c_{j s_{2}} n_{j \uparrow} n_{j \downarrow} c_{j s_{3}}^{\dagger} c_{i s_{4}} \\
= & \sum_{i j} \frac{4 i t_{i j}^{m}}{U} \boldsymbol{\alpha}_{i j}^{m} \cdot\left(\boldsymbol{S}_{i} \times \boldsymbol{S}_{j}\right) .
\end{aligned}
$$

The interaction $\mathcal{H}^{D M}$ is a factor $\alpha^{m} / t^{m}$ smaller than the usual spin-spin interaction constant $J$. While $\alpha^{m} / t^{m}$ will be less than one, it is not necessarily small.

\section{References}

1. V.M. Edelstein, Sov. Phys. JETP 68, 1244 (1989)

2. L.P. Gor'kov, E.I. Rashba, Phys. Rev. Lett. 87, 037004 (2001)

3. V. Barzykin, L.P. Gor'kov, Phys. Rev. Lett. 89, 227002 (2002)

4. O. Dimitrova, M. Feigelman, Sov. Phys. JETP Lett. 78, 637 (2003)

5. E. Bauer et al., Phys. Rev. Lett. 92, 027003 (2004)

6. P.A. Frigeri, D.F. Agterberg, A. Koga, M. Sigrist, Phys. Rev. Lett. 92, 097001 (2004)

7. P.W. Anderson, Phys. Rev. B 30, 4000 (1984)

8. L.N. Bulaevskii, A. Guseinov, A. Rusinov, Sov. Phys. JETP 44, 1243 (1975)

9. P.A. Frigeri, D.F. Agterberg, M. Sigrist, New. J. Phys. 6, $115(2004)$

10. K.V. Samokhin, Phys. Rev. Lett. 94, 027004 (2005)

11. V.P. Mineev, Phys. Rev. B 71, 012509 (2005)

12. R.P. Kaur, D.F. Agterberg, M. Sigrist, Phys. Rev. Lett. 94, $137002(2005)$

13. K.V. Samokhin, E.S. Zijlstra, S.K. Bose, Phys. Rev. B 69, 094514 (2004) [Erratum: Phys. Rev. B 70, 069902(E) (2004)]

14. I.A. Sergienko, S.H. Curnoe, Phys. Rev. B 70, 214510 (2004)

15. V.P. Mineev, Int. J. Mod. Phys. B 18, 2963 (2004)

16. G. Dresselhaus, Phys. Rev. 100, 580 (1955)

17. M. Sigrist, K. Ueda, Rev. Mod. Phys. 63, 239 (1991)

18. V.P. Mineev, K.V. Samokhin, Introduction to Unconventional Superconductivity (Gordon and Breach Science Publishers, 1999)

19. I. Dzyaloshinskii, J. Phys. Chem. Solids 4, 241 (1958)

20. T. Moriya, Phys. Rev. 120, 91 (1960) 
21. Note that the in some cases $\boldsymbol{g}_{\boldsymbol{k}}$ cannot be attributed to a single irreducible representation. In this case the representation $\Gamma_{n}^{-}$is composite containing more than one irreducible represenation. We avoid this case here, since our aim is to give a description of pairing in $\mathrm{CePt}_{3} \mathrm{Si}$ where $\Gamma_{n}^{-}$is irreducible.

22. A.A. Abrikosov, L. Gor'kov, I.E. Dzyaloshnskii (Dover, New York, 1975)

23. P.W. Anderson, J. Phys. Chem. Solids 11, 26 (1959)

24. A. Larkin, Sov. Phys. JETP Lett. 2, 130 (1965)
25. Q. Yuan et al., Phys. Rev. 68, 174510 (2003)

26. M. Yogi et al., Phys. Rev. Lett. 93, 027003 (2004)

27. M. Yogi et al., Physica B 359, 389 (2005)

28. E. Bauer et al., Physica 359, 360 (2005)

29. N. Hayashi et al., Phys. Rev. B 73, 092508 (2006)

30. K.V. Samokhin, Phys. Rev. B 72, 054514 (2005)

31. I. Bonalde, W. Braemer-Escamilla, E. Bauer, Phys. Rev. Lett. 94, 207002 (2005)

32. K. Izawa et al., Phys. Rev. Lett. 94, 197002 (2005) 